Supplementary Information

\title{
Computational Prediction of Boron-based MAX Phases and
}

\section{MXene Derivatives}

Nanxi Miao ${ }^{1,2}$, Junjie Wang ${ }^{*}, 1,2,3$, Yutong Gong ${ }^{1,2}$, Jiazhen $\mathrm{Wu}^{3}$, Haiyang $\mathrm{Niu}^{1,2}$, Shiyao Wang ${ }^{1,2}$, Kun $\mathrm{Li}^{1,2}$, Artem R. Oganov *,4,1,2, Tomofumi Tada ${ }^{3,5}$, Hideo Hosono ${ }^{3}$

1. State Key Laboratory of Solidification Processing, Northwestern Polytechnical University, Xi'an, Shaanxi 710072, People's Republic of China.

2. International Center for Materials Discovery, School of Materials Science and Engineering, Northwestern Polytechnical University, Xi'an, Shaanxi 710072, People's Republic of China.

3. Materials Research Center for Element Strategy, Tokyo Institute of Technology, 4259 Nagatsuta-cho, Midori-ku, Yokohama, Kanagawa 226-8503, Japan.

4. Skolkovo Innovation Center, Skolkovo Institute of Science and Technology, 3 Nobel Steet, Moscow 143026, Russia.

5. Kyushu University Platform of Inter/Transdisciplinary Energy Research, Kyushu University, 744 Motooka, Nishi-ku, Fukuoka 819-0395, Japan 


\section{Settings for the Calculations of Adsorption and Diffusion Behaviors on 2D Borides}

The adsorption energies of functional groups $T=\mathrm{OH}, \mathrm{H}, \mathrm{Cl}, \mathrm{F}$, or $\mathrm{O}$ on $2 \mathrm{D} \mathrm{M}_{2} \mathrm{~B}, \mathrm{M}_{2} \mathrm{~B}_{2}$ and $\mathrm{M}_{3} \mathrm{~B}_{4}$ $(\mathrm{M}=\mathrm{Hf}, \mathrm{Zr})$ were calculated using the following formula,

$E_{a d}=\frac{\left(E_{M B T}-E_{M B}-\lambda / 2 E_{T 2}\right)}{\lambda}$

where $E_{M B T}$ is the total energy of the boride MXene functionalized with $T=\mathrm{OH}, \mathrm{H}, \mathrm{Cl}, \mathrm{F}$, or $\mathrm{O}, E_{M B}$ represents the total energy of the pristine boride MXene, $E_{T}$ denotes the energy of a functional group in its ground state. For $\mathrm{H}, \mathrm{Cl}, \mathrm{F}$ and $\mathrm{O}$ groups, $E_{T}$ is the half of $\mathrm{T}_{2}$ molecule in a cubic supercell of

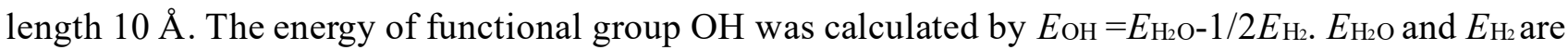
the calculated energies of isolated $\mathrm{H}_{2} \mathrm{O}$ and $\mathrm{H}_{2}$ molecules, respectively.

We performed full geometry optimizations for the $\mathrm{Li}^{+} / \mathrm{Na}^{+}$-incorporated MXenes using a $4 \times 4 \times 1$ supercell for $\mathrm{M}_{2} \mathrm{BA}_{2}$ and a $3 \times 3 \times 1$ supercell for $\mathrm{M}_{2} \mathrm{~B}_{2} \mathrm{~A}_{2}\left(\mathrm{~A}=\mathrm{Li}^{+}\right.$or $\left.\mathrm{Na}^{+}\right)$. The average adsorption energies $\left(E_{\text {ave }}\right)$ for the adsorption of the $\mathrm{n}^{\text {th }} \mathrm{Li}^{+} / \mathrm{Na}^{+}$layer on MXenes were calculated as:

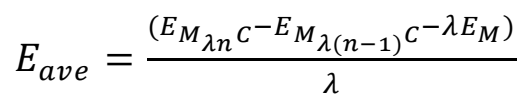

where $E_{M_{\lambda n} C}$ and $E_{M_{\lambda(n-1)} C}$ are respectively the total energies for the adsorption of $n$ and (n-1) layers of $\mathrm{Li}^{+} / \mathrm{Na}^{+}$on a MXene; $\lambda$ represents the number of the adsorbed $\mathrm{Li}^{+} / \mathrm{Na}^{+}$on $\mathrm{MXene}$ surface.

The theoretical gravimetric capacity (C) of $\mathrm{Li}^{+} / \mathrm{Na}^{+}$intercalated MXene can be estimated by the following formula,

$C=\frac{n F}{M_{M X e n e^{+}+n M_{A}}}$

where $n$ is the maximum number of adsorptions, $F$ is the Faraday constant $(26,801 \mathrm{mAh} / \mathrm{mol}), M$ MXene and $M_{\mathrm{A}}$ are the molar weights of $\mathrm{MXene}$ and $\mathrm{Li}^{+} / \mathrm{Na}^{+}$, respectively. 


\section{Supporting Figures}

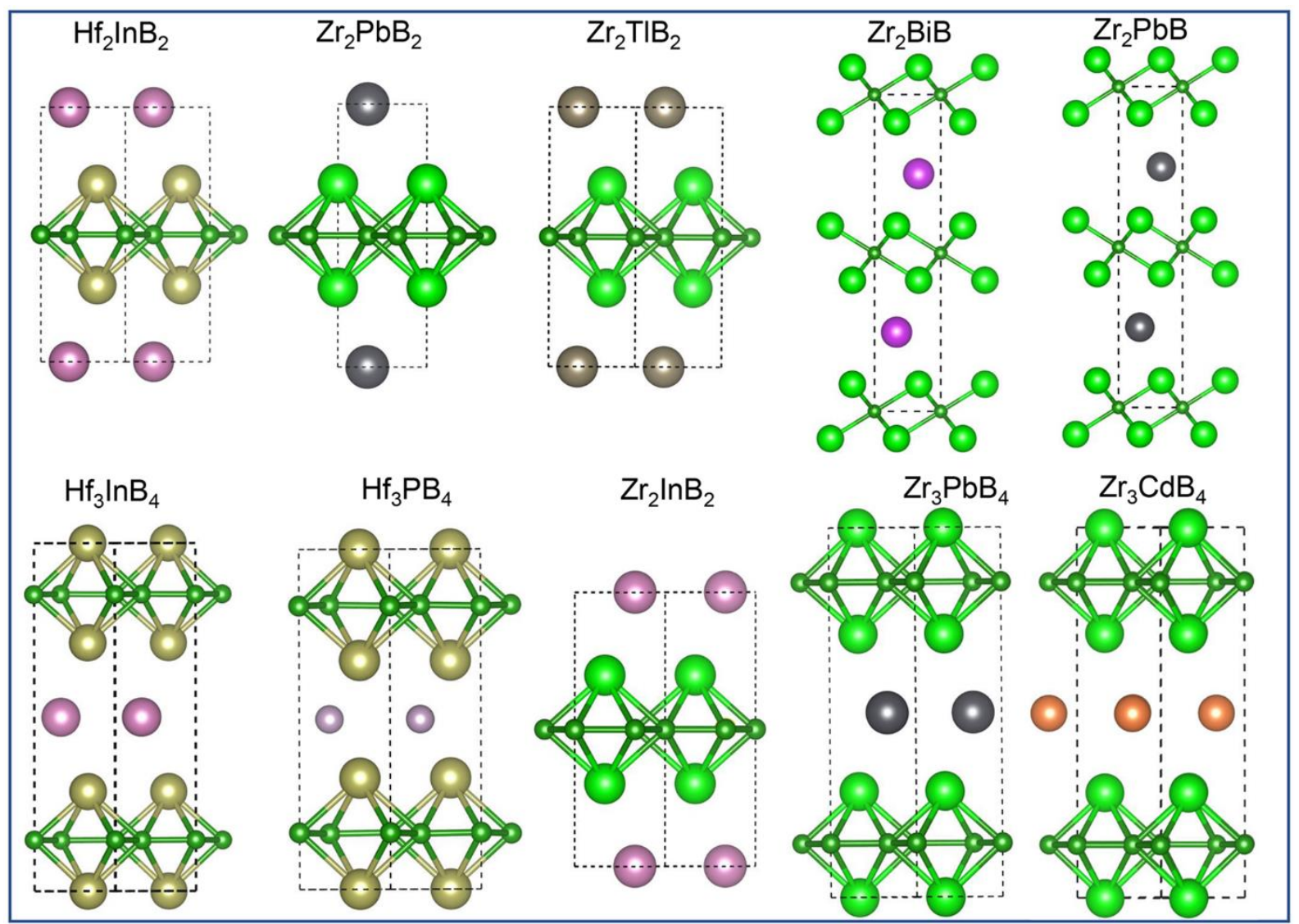

Figure S1. Crystal structures of predicted layered boron-based MAX phases with compositions of $\mathrm{M}_{2} \mathrm{AB}, \mathrm{M}_{2} \mathrm{AB}_{2}$, and $\mathrm{M}_{3} \mathrm{AB}_{4}$.
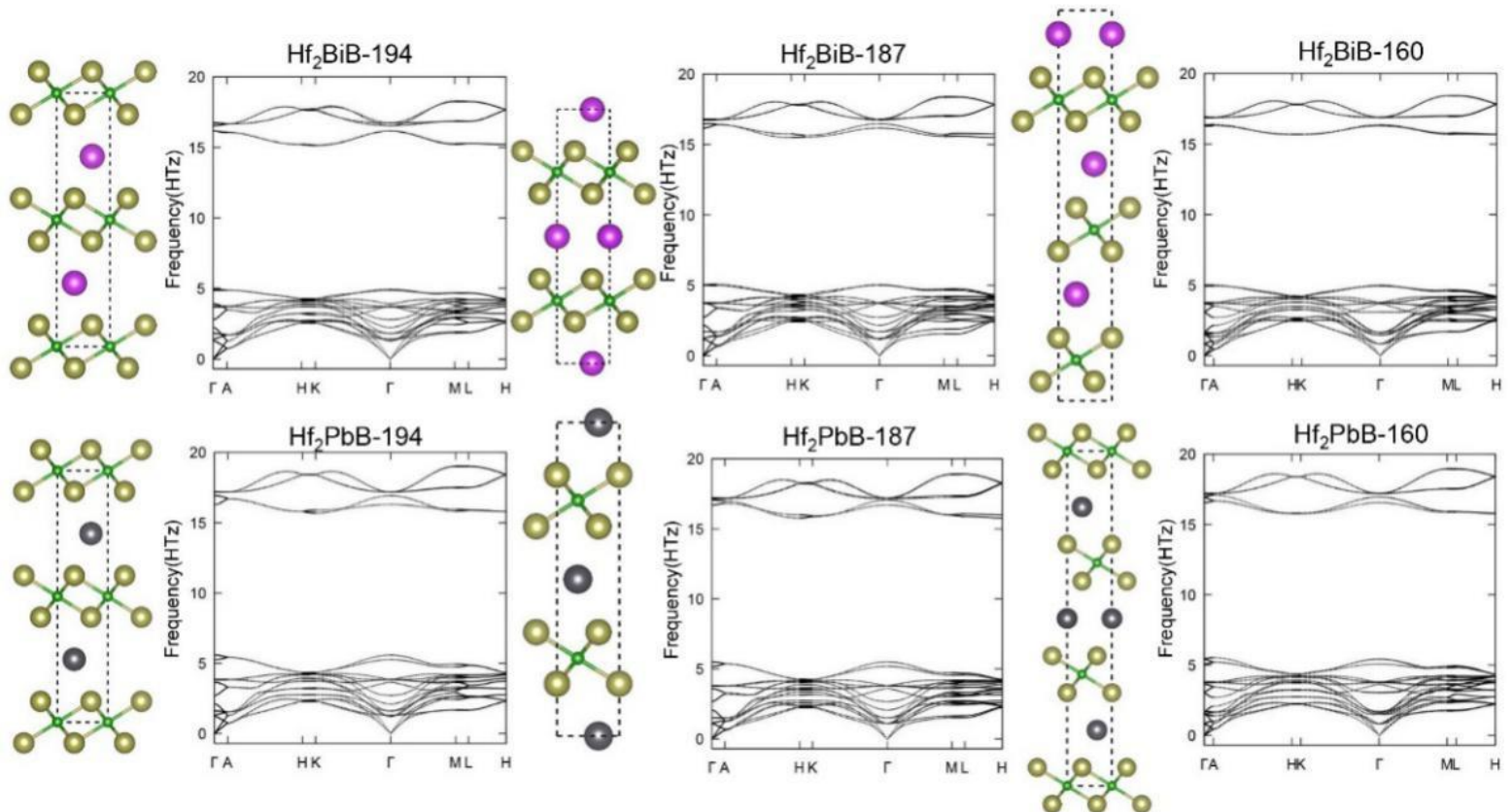

Figure S2. Calculated phonon spectra and crystal structures of $\mathrm{Hf}_{2} \mathrm{BiB}\left(\mathrm{PG}_{3} /\right.$ $m m c(N o .194), \quad P \overline{6} m 2(N o .187)$ and $R 3 m(N o .160))$ as well as $\mathrm{Hf}_{2} \mathrm{PbB}\left(\mathrm{Pb}_{3} /\right.$ $m m c(N o .194)$, P $\overline{6} m 2$ (No.187), and $R 3 m(N o .160)$ ). 

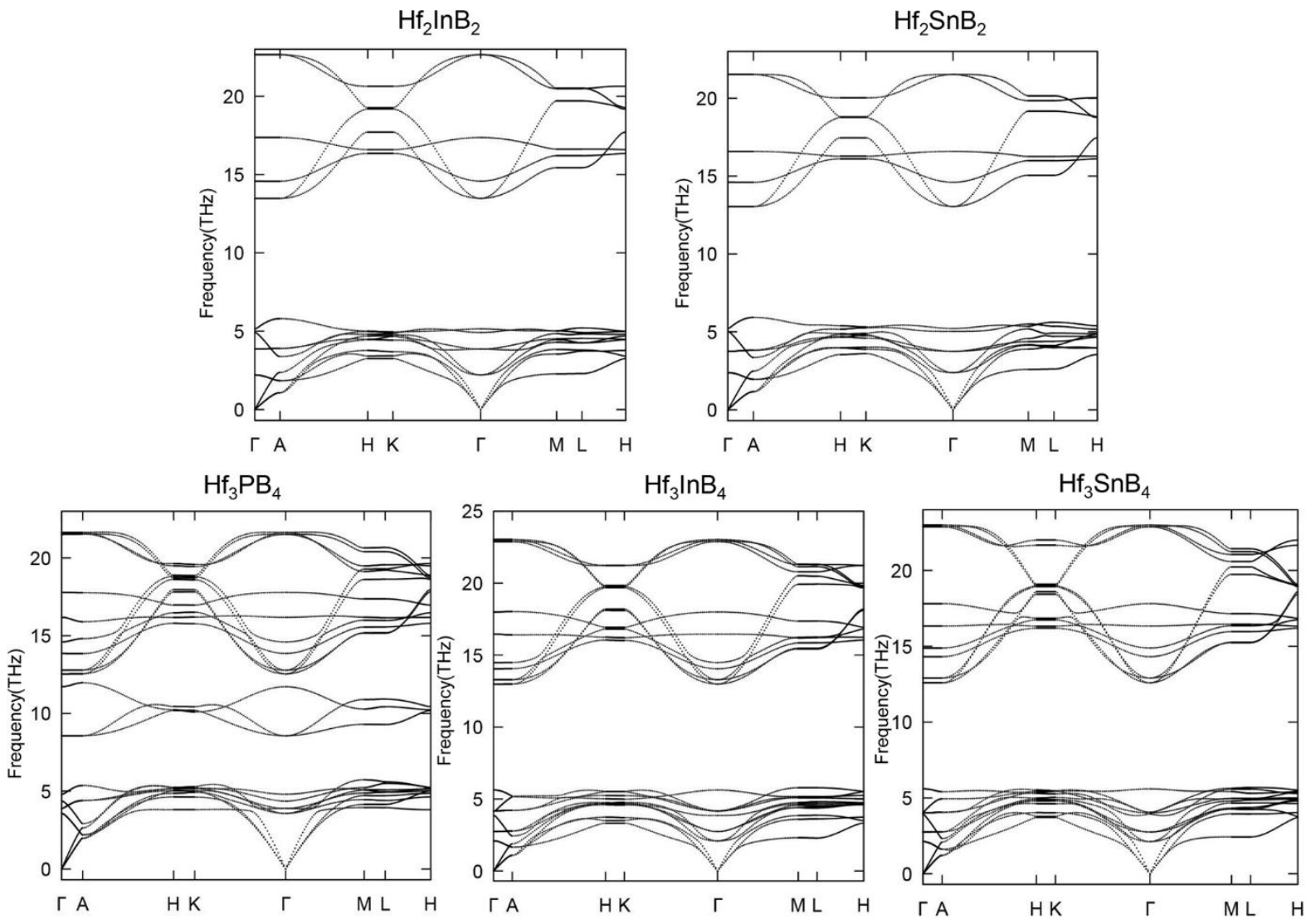

Figure S3. Computed phonon spectra of $\mathrm{Hf}_{2} \mathrm{InB}_{2}, \mathrm{Hf}_{2} \mathrm{SnB}_{2}, \mathrm{Hf}_{3} \mathrm{~PB}_{4}, \mathrm{Hf}_{3} \mathrm{InB}_{4}$, and $\mathrm{Hf}_{3} \mathrm{SnB}_{4}$ with space group $P \overline{6} m 2$ (No.187).
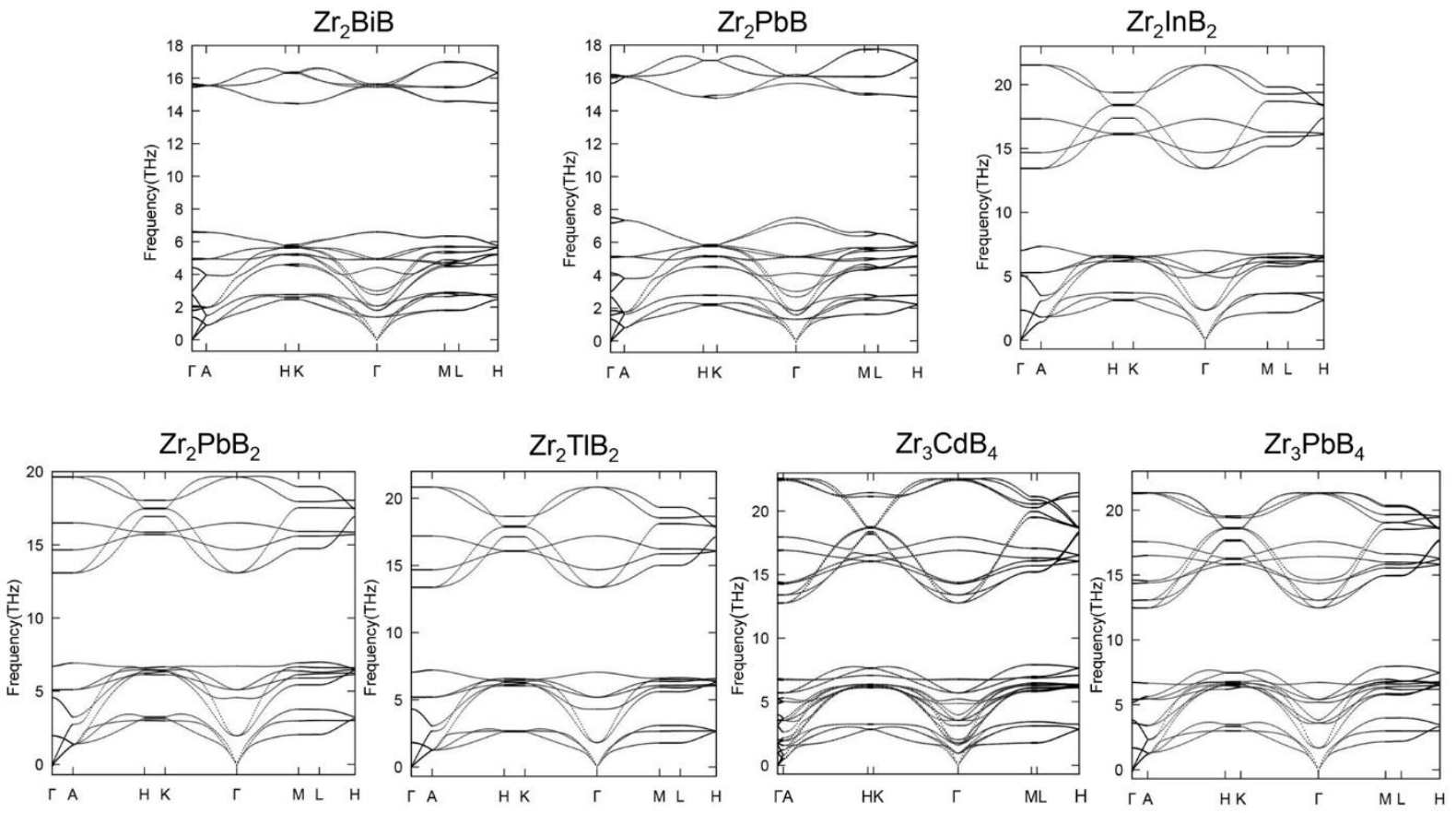

Figure S4. Computed phonon spectra of $\mathrm{Zr}_{2} \mathrm{BiB}$ and $\mathrm{Zr}_{2} \mathrm{PbB}$ with space group $\mathrm{P63} / \mathrm{mmc}$ (No. 194), and $\mathrm{Zr}_{2} \mathrm{InB}_{2}, \mathrm{Zr}_{2} \mathrm{PbB}_{2}, \mathrm{Zr}_{2} \mathrm{TlB}_{2}, \mathrm{Zr}_{3} \mathrm{CdB}_{4}$, and $\mathrm{Zr}_{3} \mathrm{PbB}_{4}$ with space group $P \overline{6} m 2$ (No. 187). 


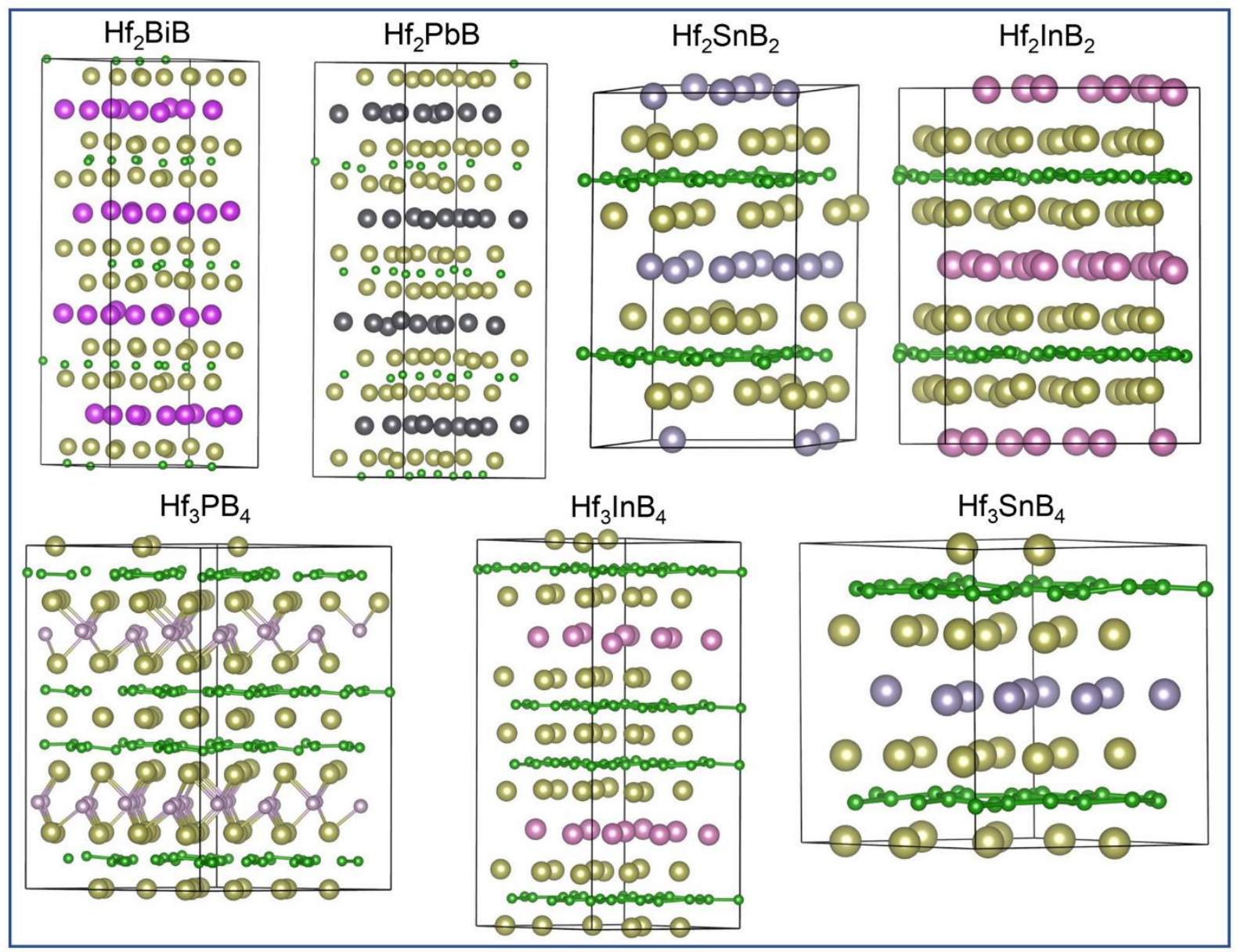

Figure S5. Equilibrium structures of predicted boron-based MAX phases in Hf-A-B system at $900 \mathrm{~K}$ after a 10 ps AIMD simulation.

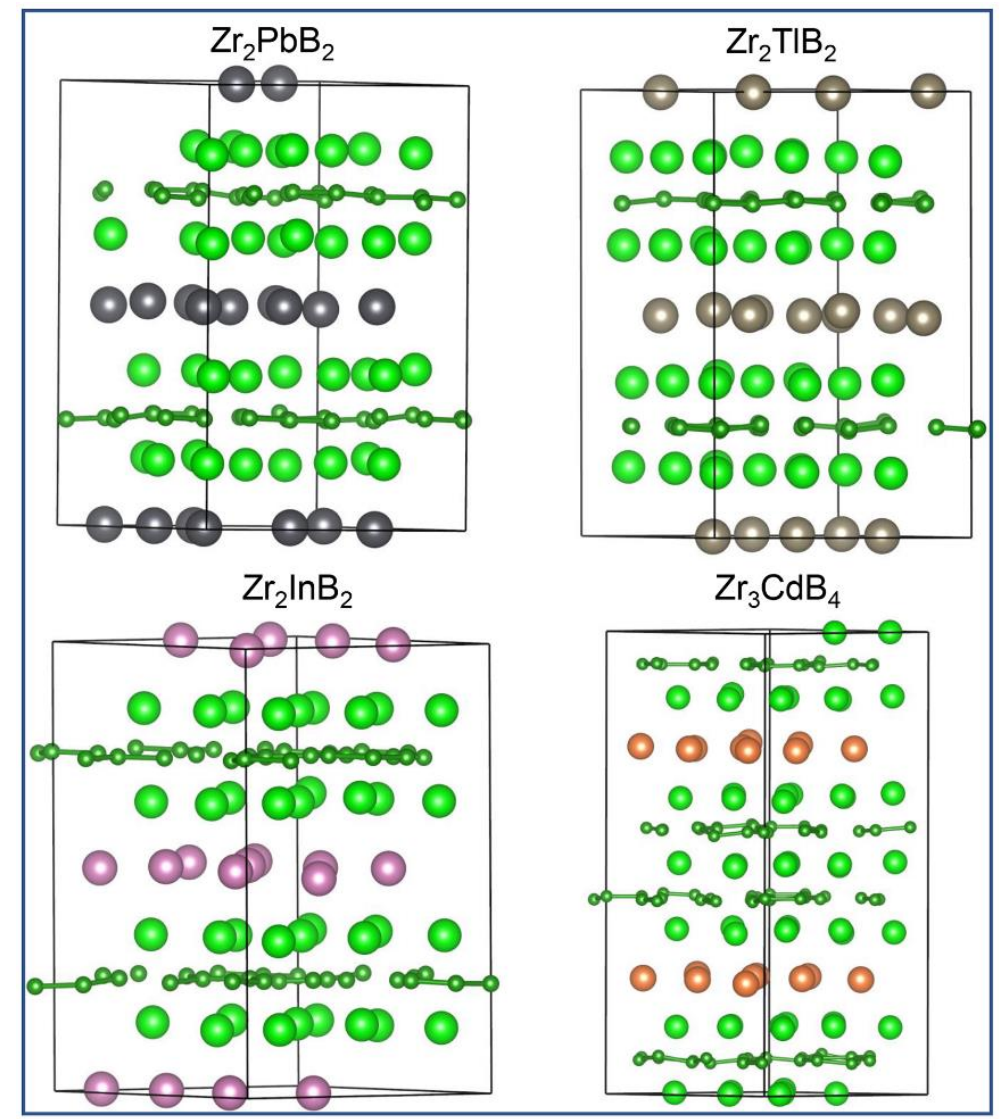

Figure S6. Equilibrium structures of predicted boron-based MAX phases in Zr-A-B system at $900 \mathrm{~K}$ after a 10 ps AIMD simulation. 

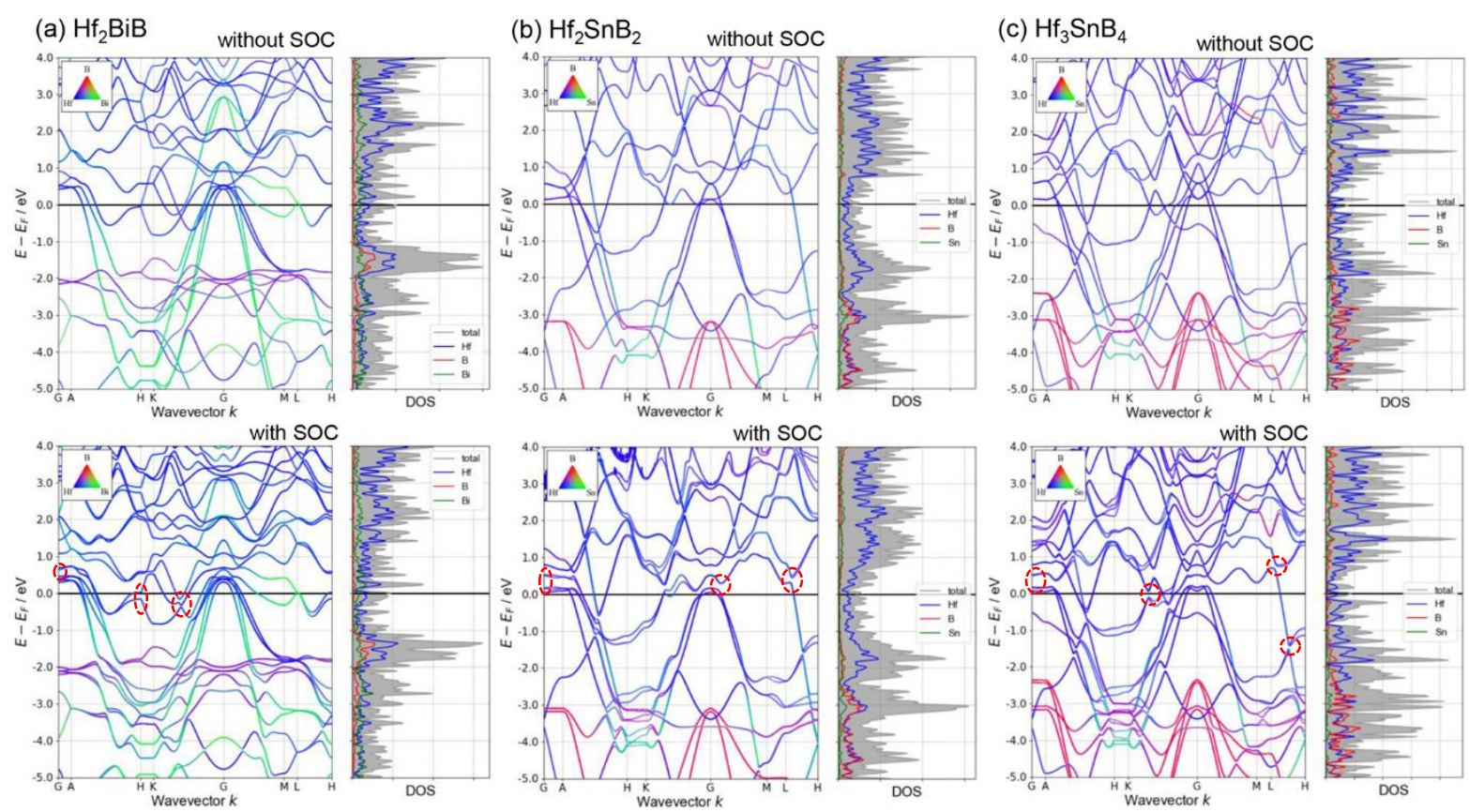

Figure S7. Calculated projected band structures and density of states for (a) $\mathrm{Hf}_{2} \mathrm{BiB}$, (b) $\mathrm{Hf}_{2} \mathrm{SnB}_{2}$, and (c) $\mathrm{Hf}_{3} \mathrm{SnB}_{4}$ phases without (top) and with (bottom) relativistic spin-orbit coupling (the red circle highlighted the opened band gap).

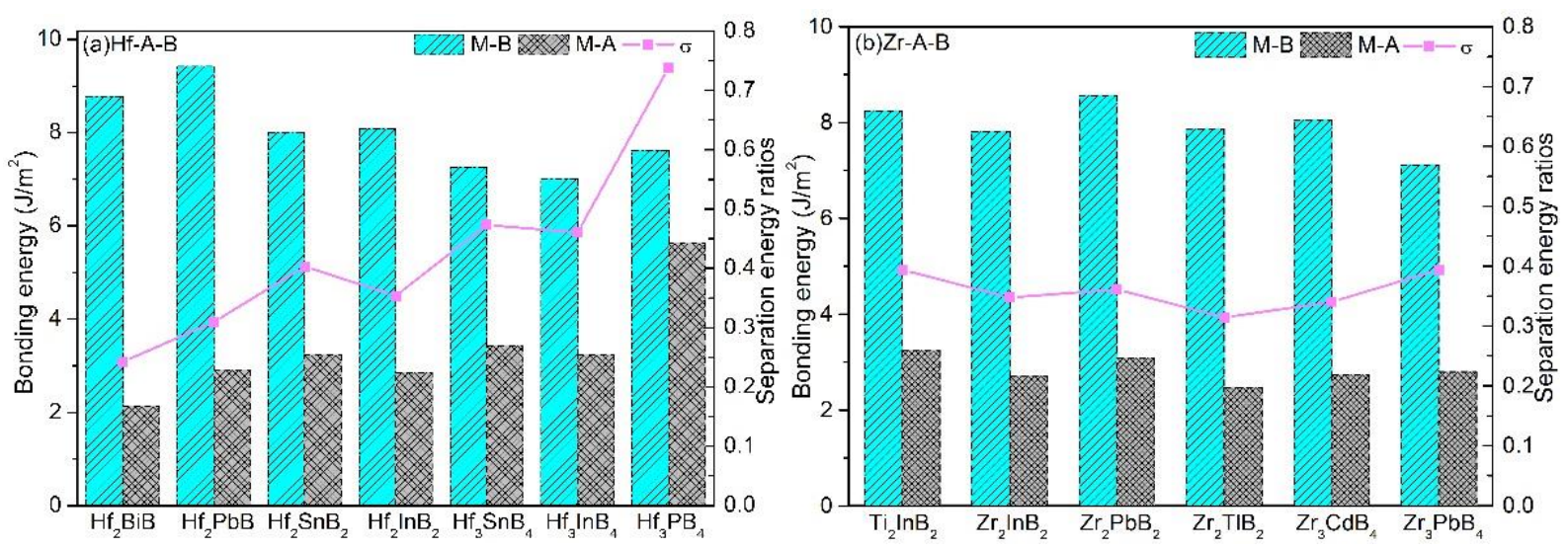

Figure S8. Calculated bonding energies and separation energy ratios $(\sigma)$ at different interfaces of new MAX phases in (a) Hf-A-B system and (b) Zr-A-B system. 


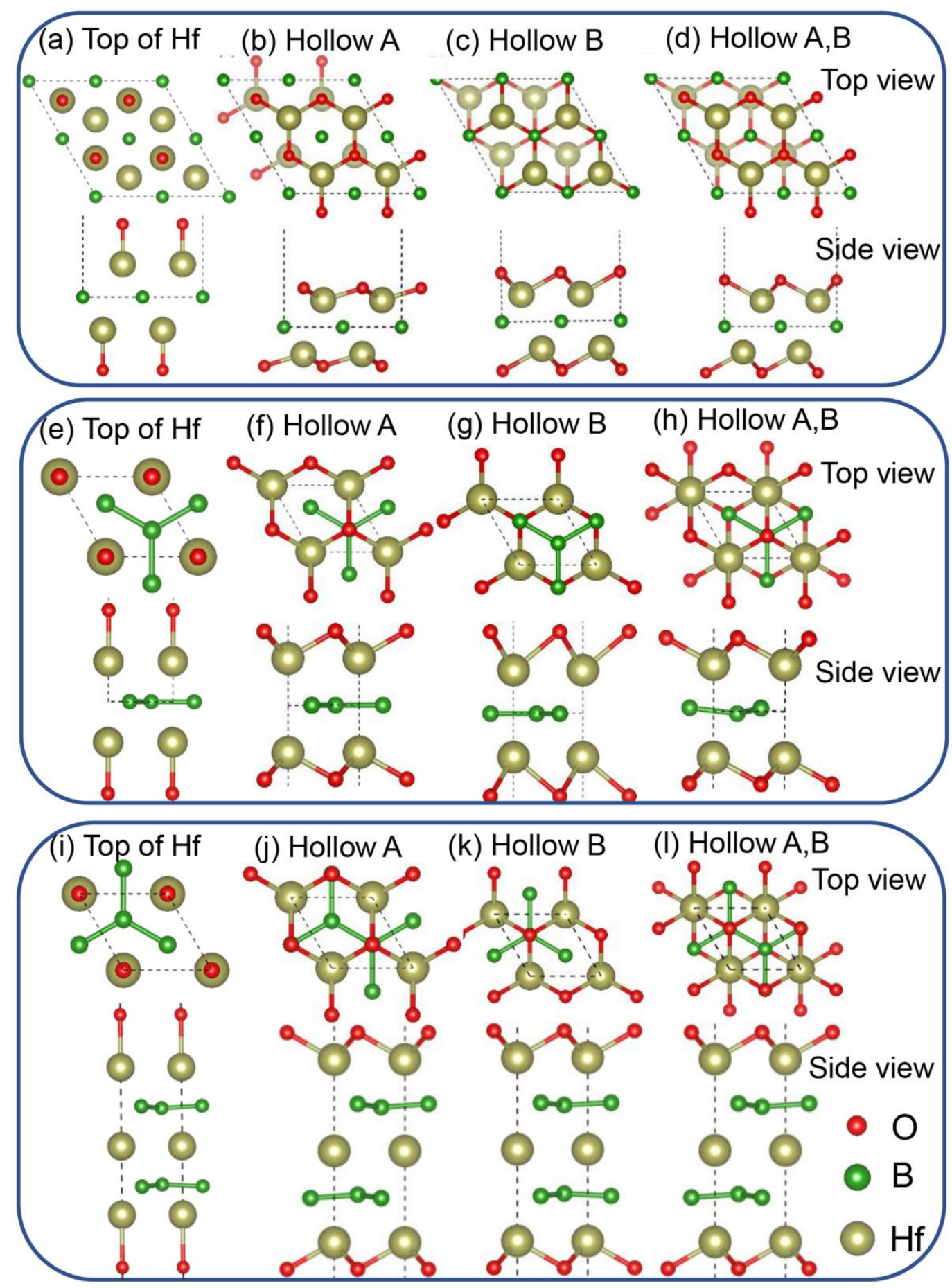

Figure S9. Top and side views of different configuration models of $2 \mathrm{D}$ borides with functional groups $T\left(T=\mathrm{O}, \mathrm{H}, \mathrm{F}, \mathrm{Cl}\right.$, and $\mathrm{OH}$ ) (a-d) $\mathrm{Hf}_{2} \mathrm{BT} 2$; (e-h) $\mathrm{Hf}_{2} \mathrm{~B}_{2} \mathrm{~T}_{2}$ or $\mathrm{Zr}_{2} \mathrm{~B}_{2} \mathrm{~T}_{2}$, and (i-l) $\mathrm{Hf}_{3} \mathrm{~B}_{4} \mathrm{~T}_{2}$ or $\mathrm{Zr}_{3} \mathrm{~B}_{4} \mathrm{~T}_{2}$. Three kinds of stable adsorption sites (Top, Hollow A, and B) were considered in the construction of these configurations. 

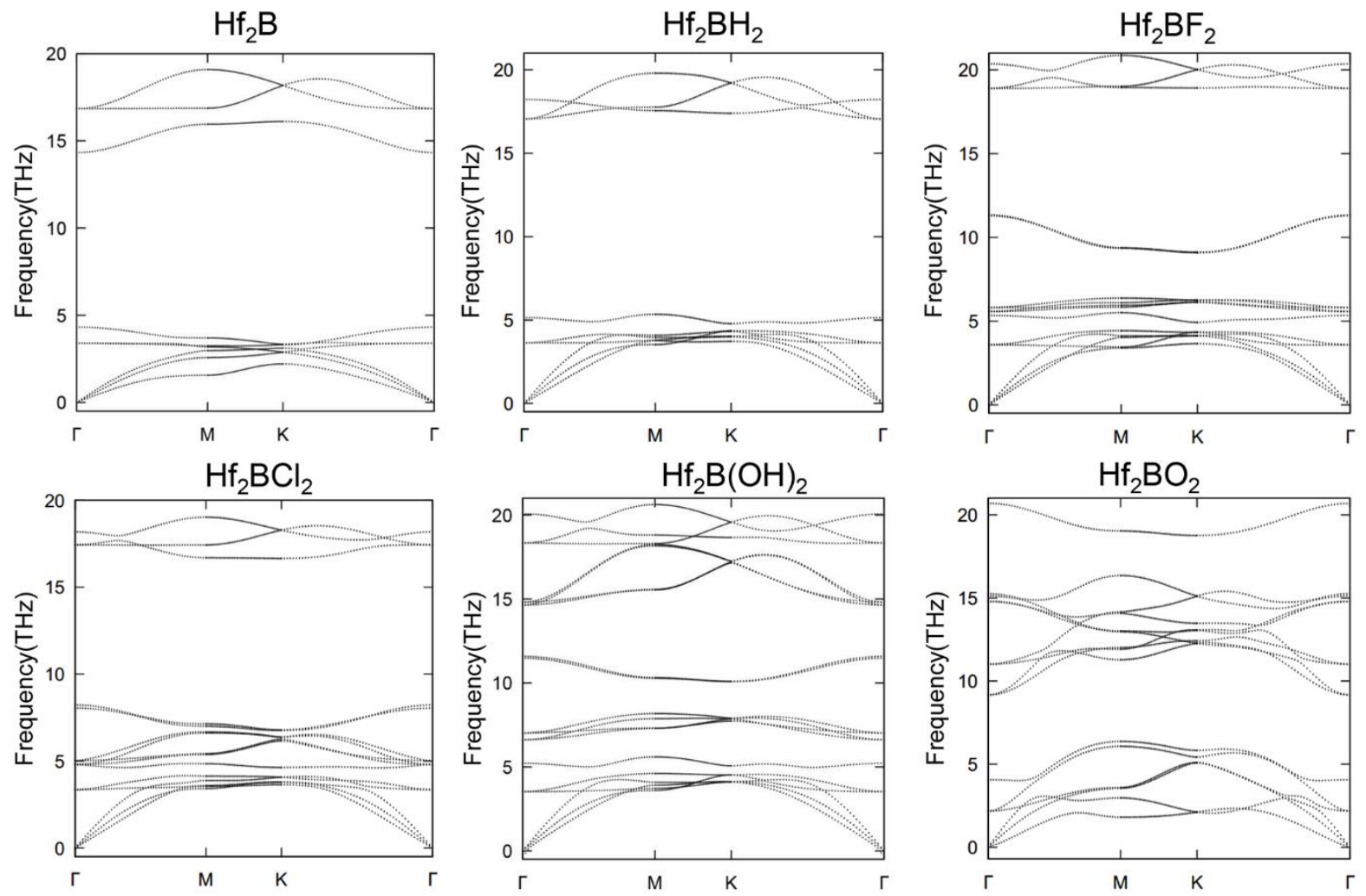

Figure S10. Computed phonon spectra of the $2 \mathrm{D} \mathrm{Hf}_{2} \mathrm{~B}$ with functional groups $T(T=\mathrm{H}, \mathrm{F}, \mathrm{Cl}, \mathrm{OH}$, and O).
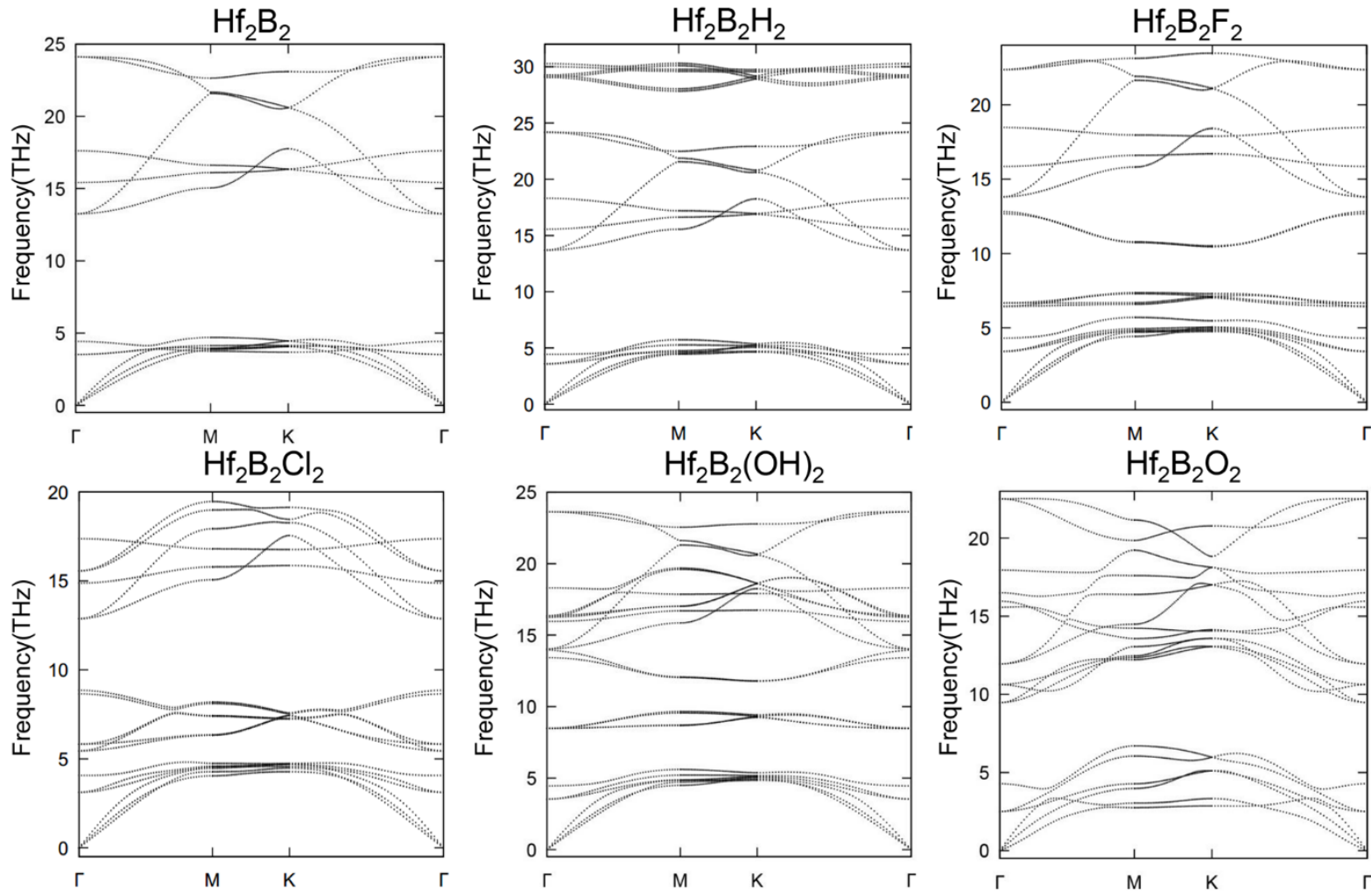

Figure S11. Computed phonon spectra of the $2 \mathrm{D} \mathrm{Hf}_{2} \mathrm{~B}_{2}$ with functional groups $T(T=\mathrm{H}, \mathrm{F}, \mathrm{Cl}, \mathrm{OH}$, and O). 

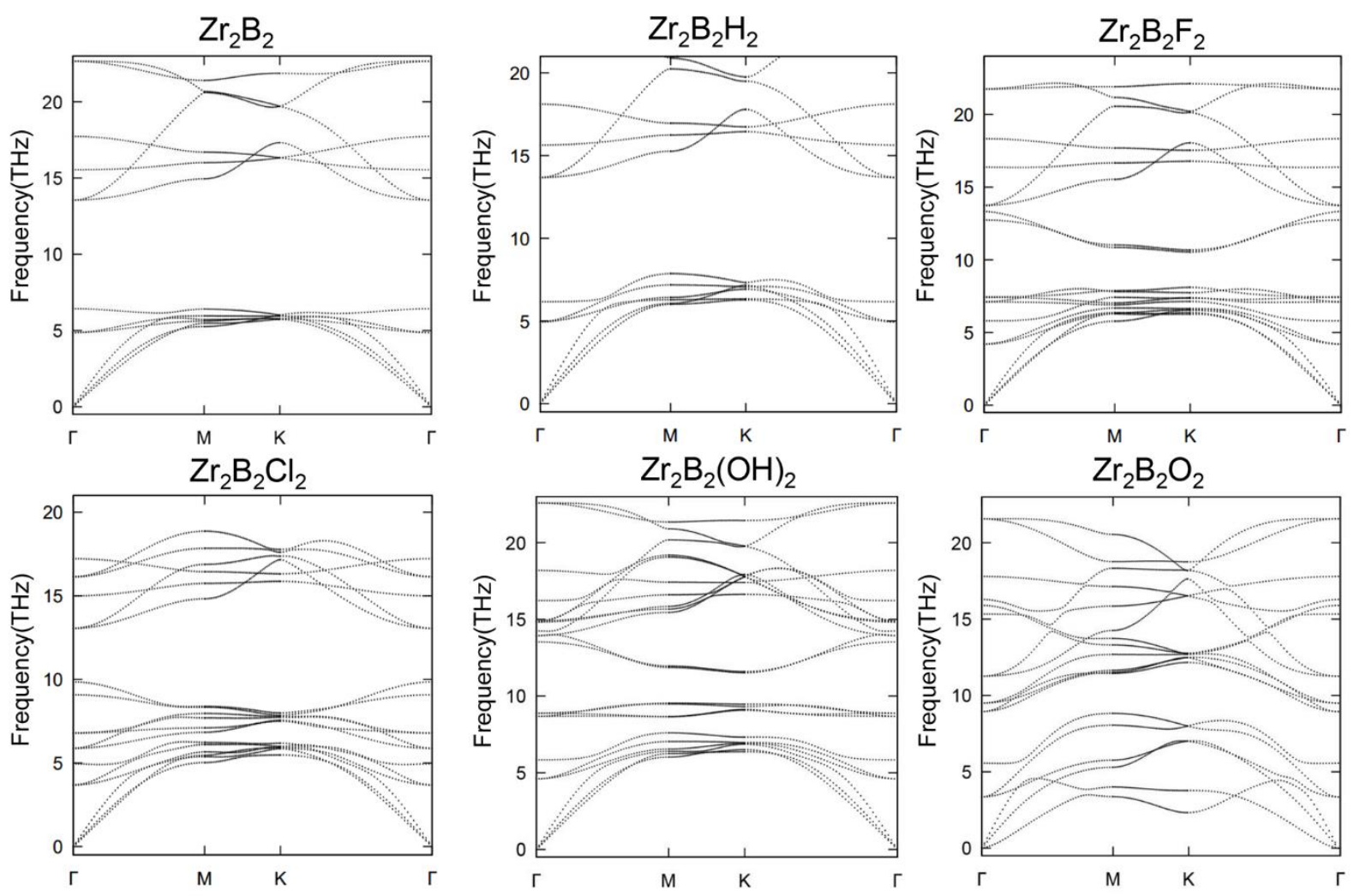

Figure S12. Computed phonon spectra of the $2 \mathrm{D} \mathrm{Zr}_{2} \mathrm{~B}_{2}$ with functional groups $T(T=\mathrm{H}, \mathrm{F}, \mathrm{Cl}, \mathrm{OH}$, and O).
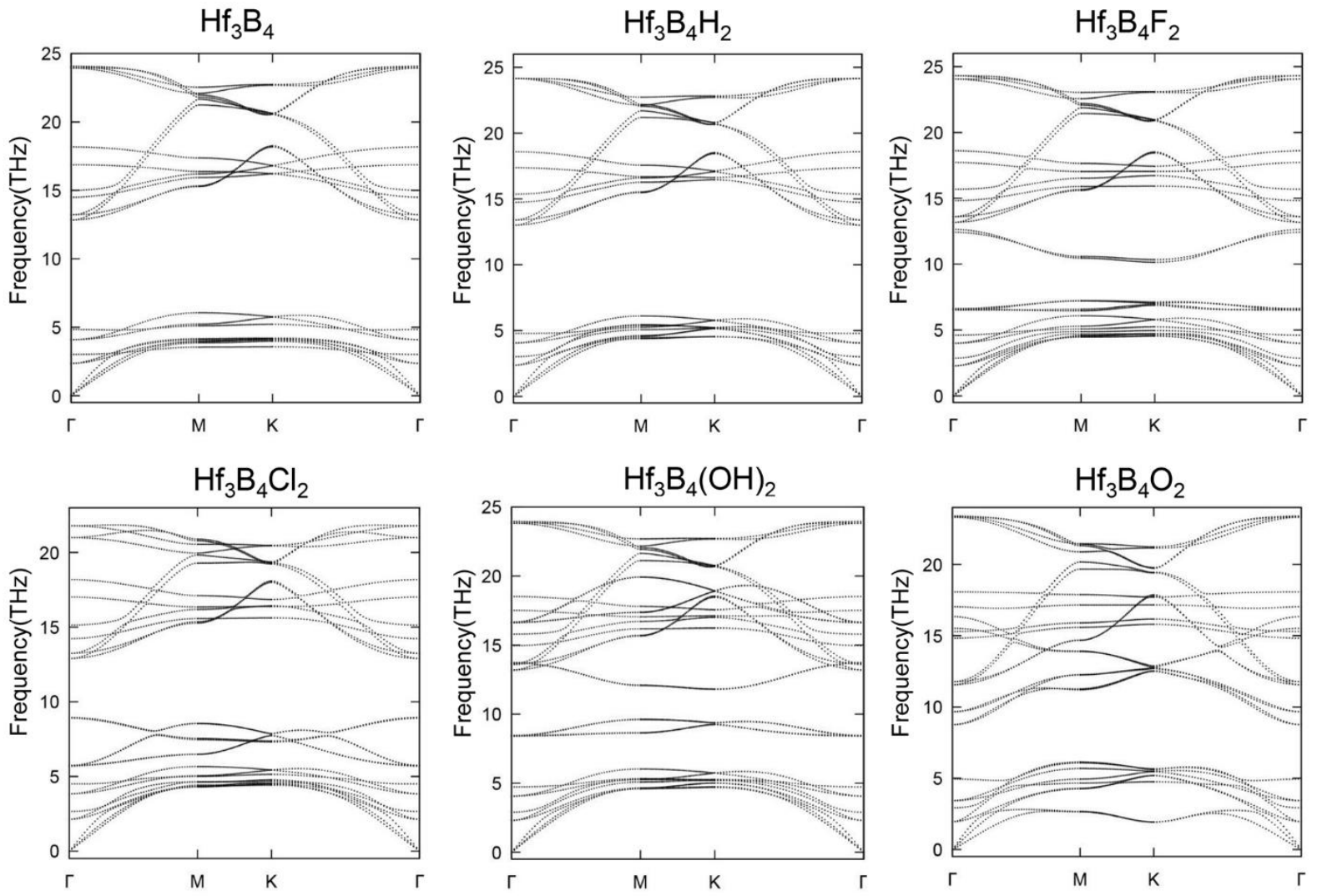

Figure S13. Computed phonon spectra of the $2 \mathrm{D} \mathrm{Hf}_{3} \mathrm{~B}_{4}$ with functional groups $T(T=\mathrm{H}, \mathrm{F}, \mathrm{Cl}, \mathrm{OH}$, and $\mathrm{O})$. 

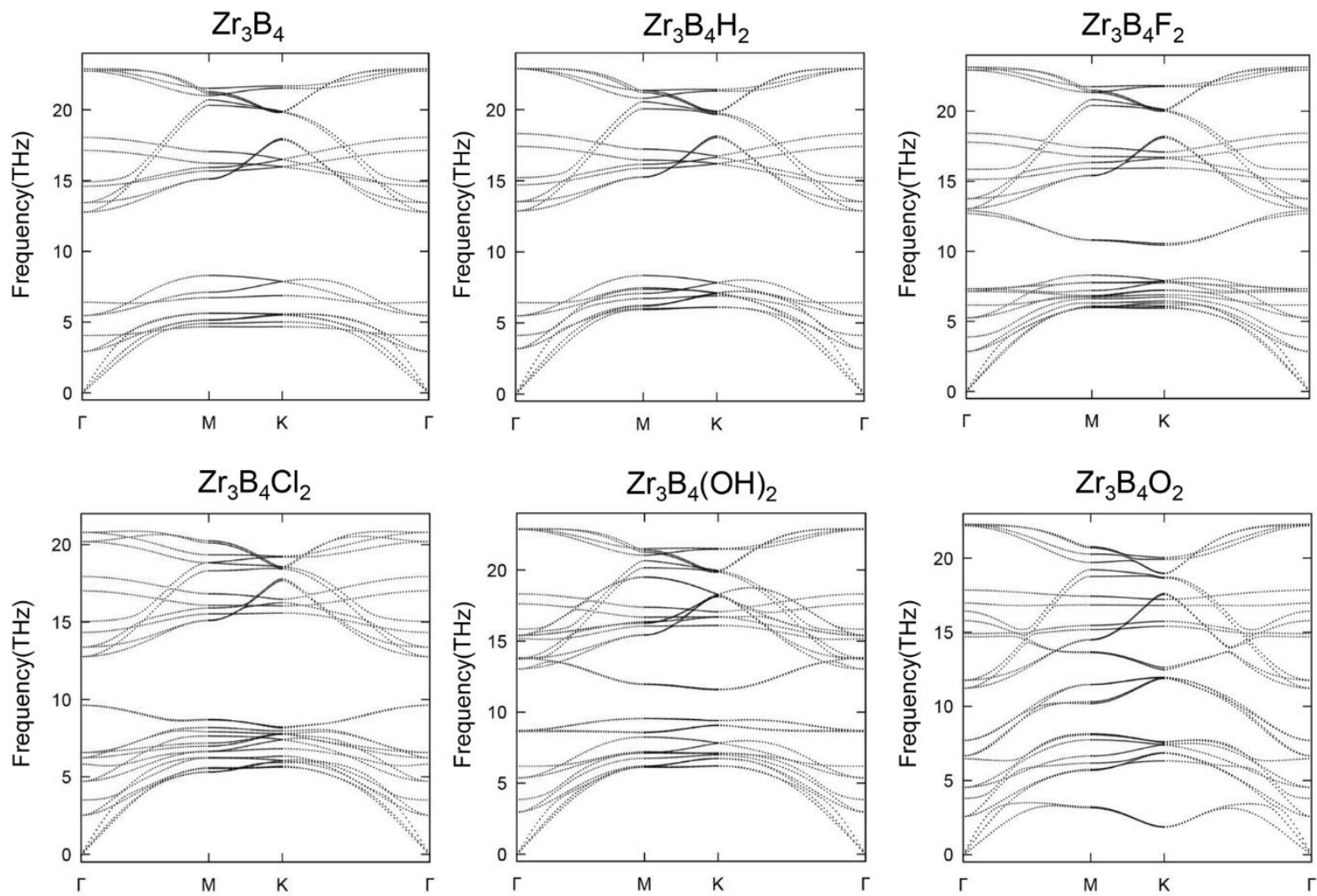

Figure S14. Computed phonon spectra of the $2 \mathrm{D} \mathrm{Zr}_{3} \mathrm{~B}_{4}$ with and without functional groups $T(T=\mathrm{H}$, $\mathrm{F}, \mathrm{Cl}, \mathrm{OH}$, and $\mathrm{O}$ ).

$\mathrm{Hf}_{2} \mathrm{~B}$

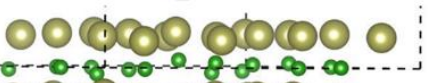
000000000

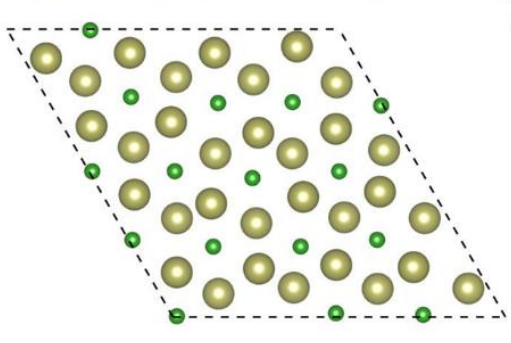

$\mathrm{Hf}_{3} \mathrm{~B}_{4}$
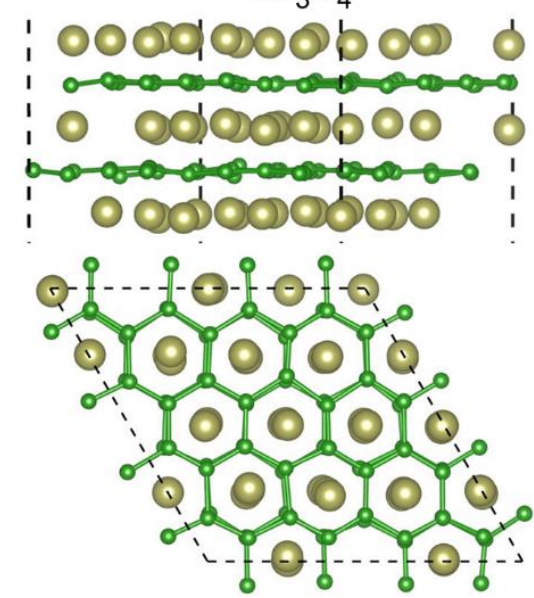

$\mathrm{Hf}_{2} \mathrm{~B}_{2}$

- 08000ب000

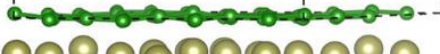
00000000000

ia a a a

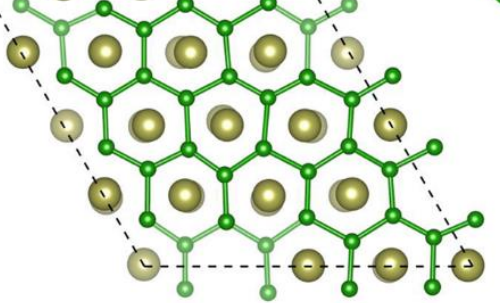

$\mathrm{Zr}_{2} \mathrm{~B}_{2}$

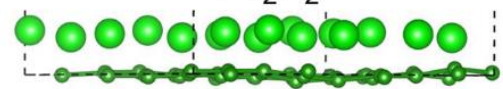

- 000000000

$\mathrm{C}^{\mathrm{a}-\mathrm{O}} \mathrm{a}-\mathrm{O}$

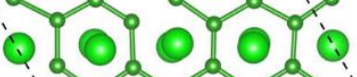

¿'lol.

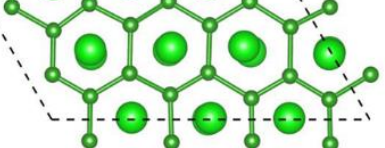

$\mathrm{Zr}_{3} \mathrm{~B}_{4}$
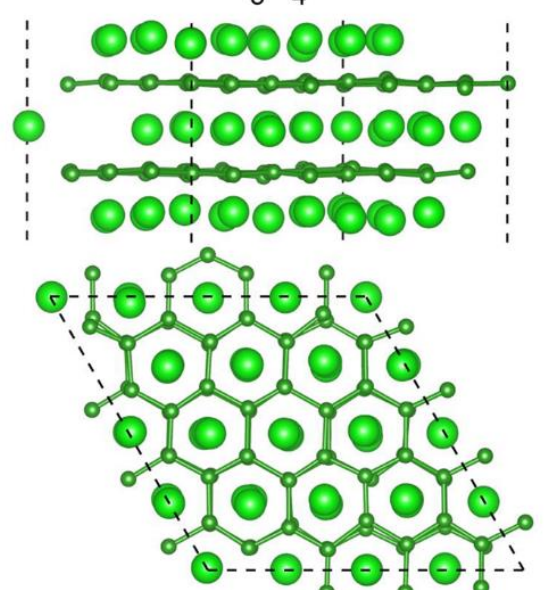

Figure S15. Equilibrium structures of predicted boride MXenes at $900 \mathrm{~K}$ after a 10 ps AIMD simulation. 

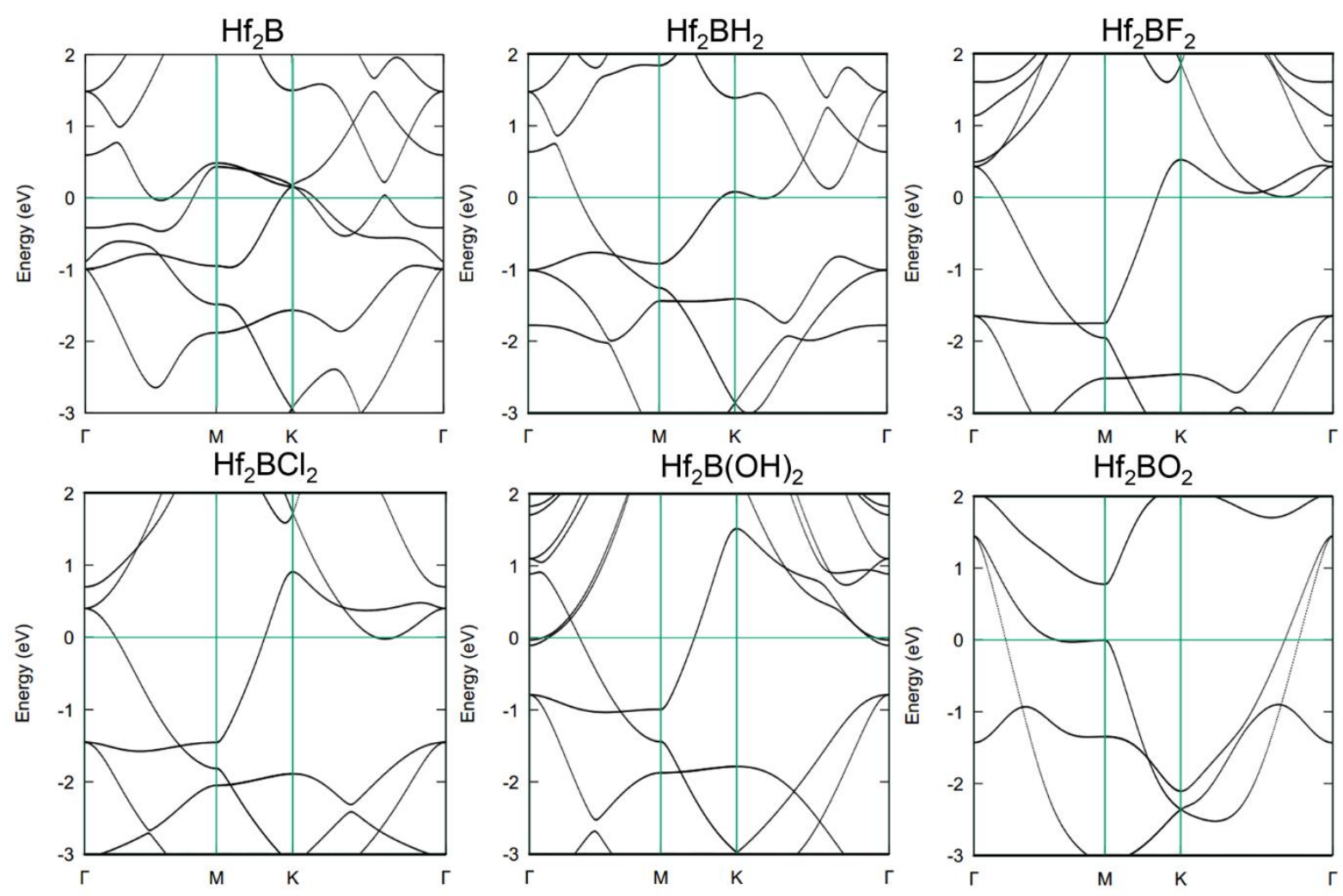

Figure S16. Calculated band structures of the $2 \mathrm{D} \mathrm{Hf}_{2} \mathrm{~B}$ with and without functional groups $T(T=\mathrm{H}, \mathrm{F}$, $\mathrm{Cl}, \mathrm{OH}$, and $\mathrm{O}$ ).
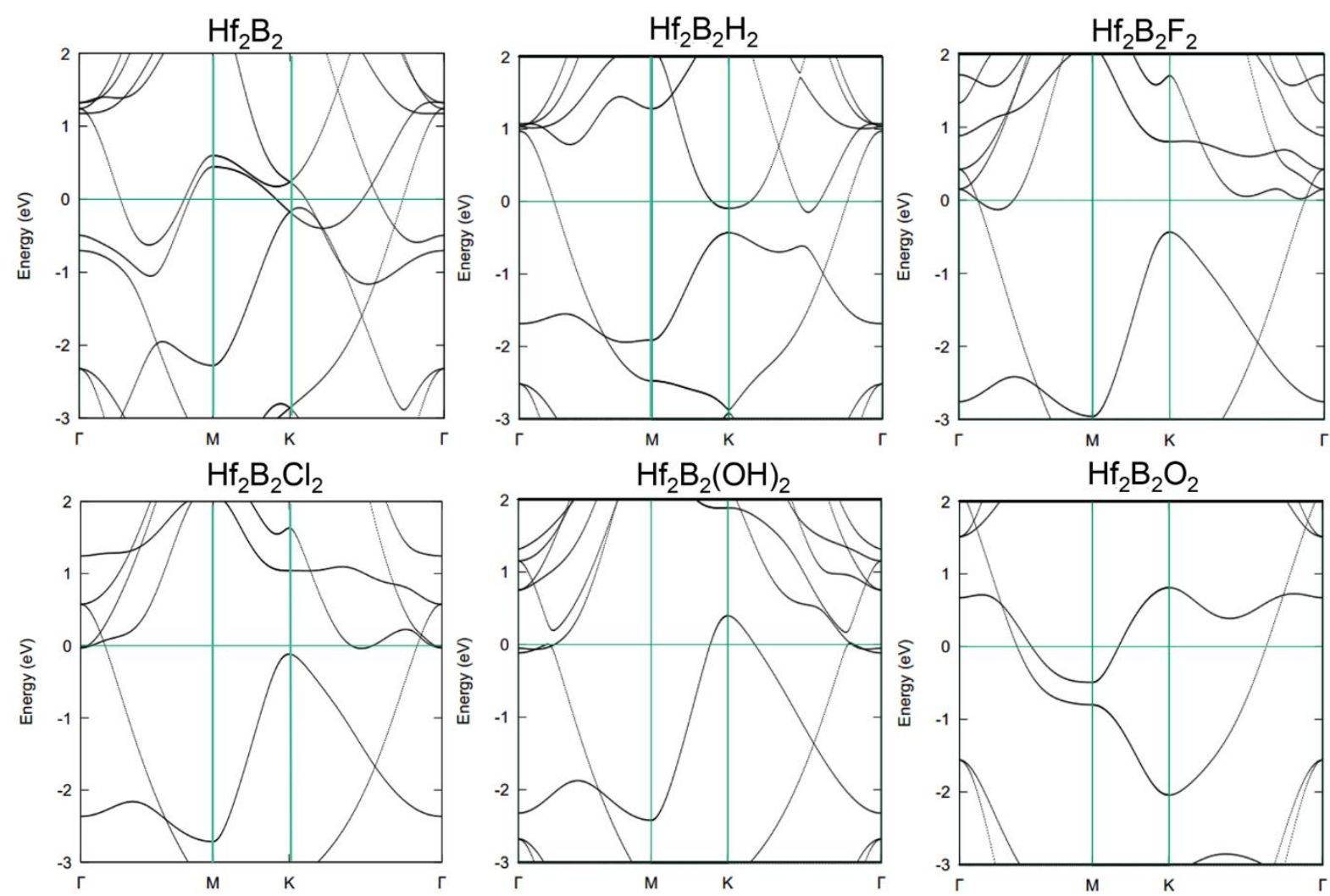

Figure S17. Calculated band structures of the $2 \mathrm{D} \mathrm{Hf}_{2} \mathrm{~B}_{2}$ with and without functional groups $T(T=\mathrm{H}$, $\mathrm{F}, \mathrm{Cl}, \mathrm{OH}$, and $\mathrm{O})$. 

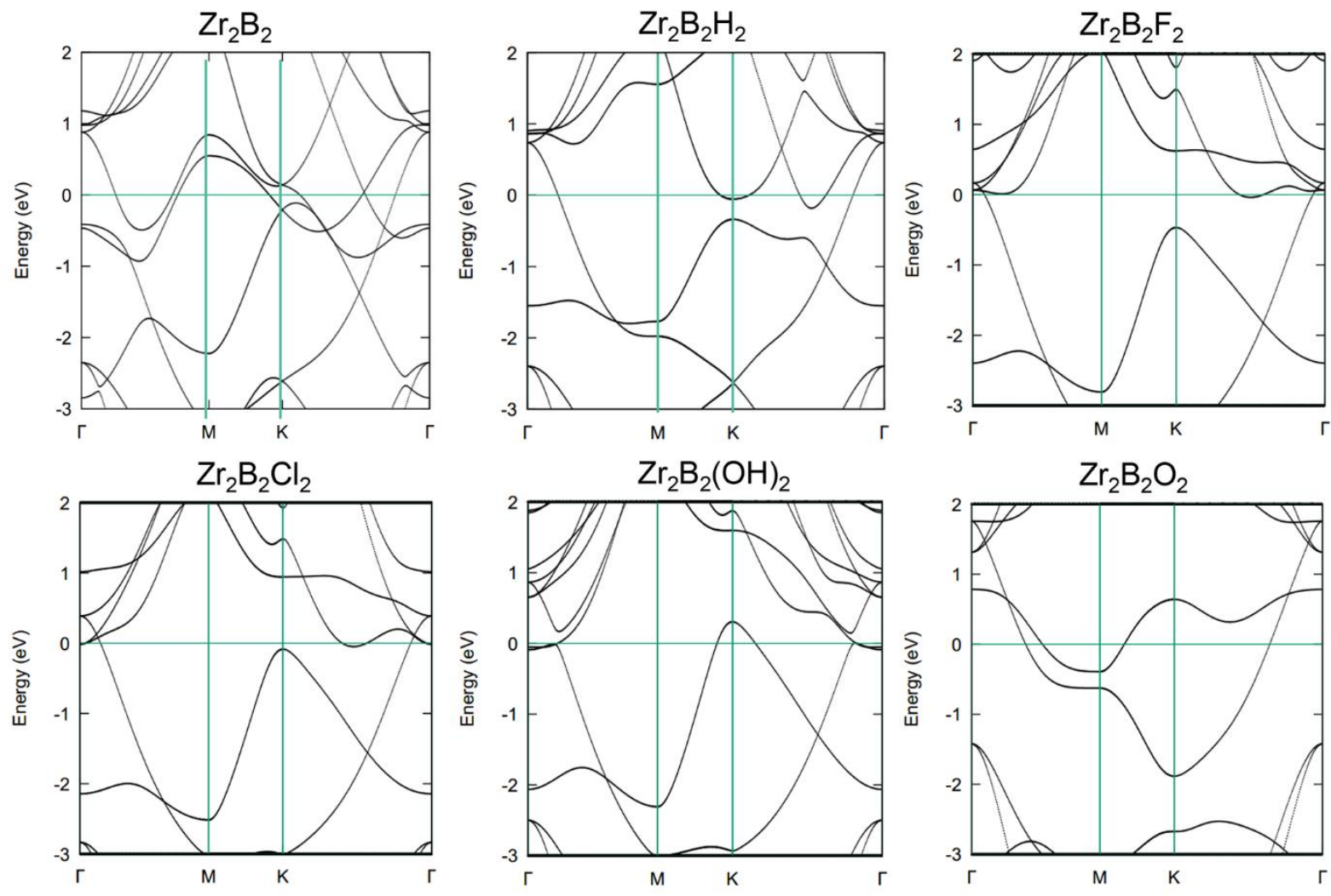

Figure S18. Calculated band structures of the $2 \mathrm{D} \mathrm{Zr}_{2} \mathrm{~B}_{2}$ with and without functional groups $T$ ( $T=\mathrm{H}$, $\mathrm{F}, \mathrm{Cl}, \mathrm{OH}$, and $\mathrm{O})$.
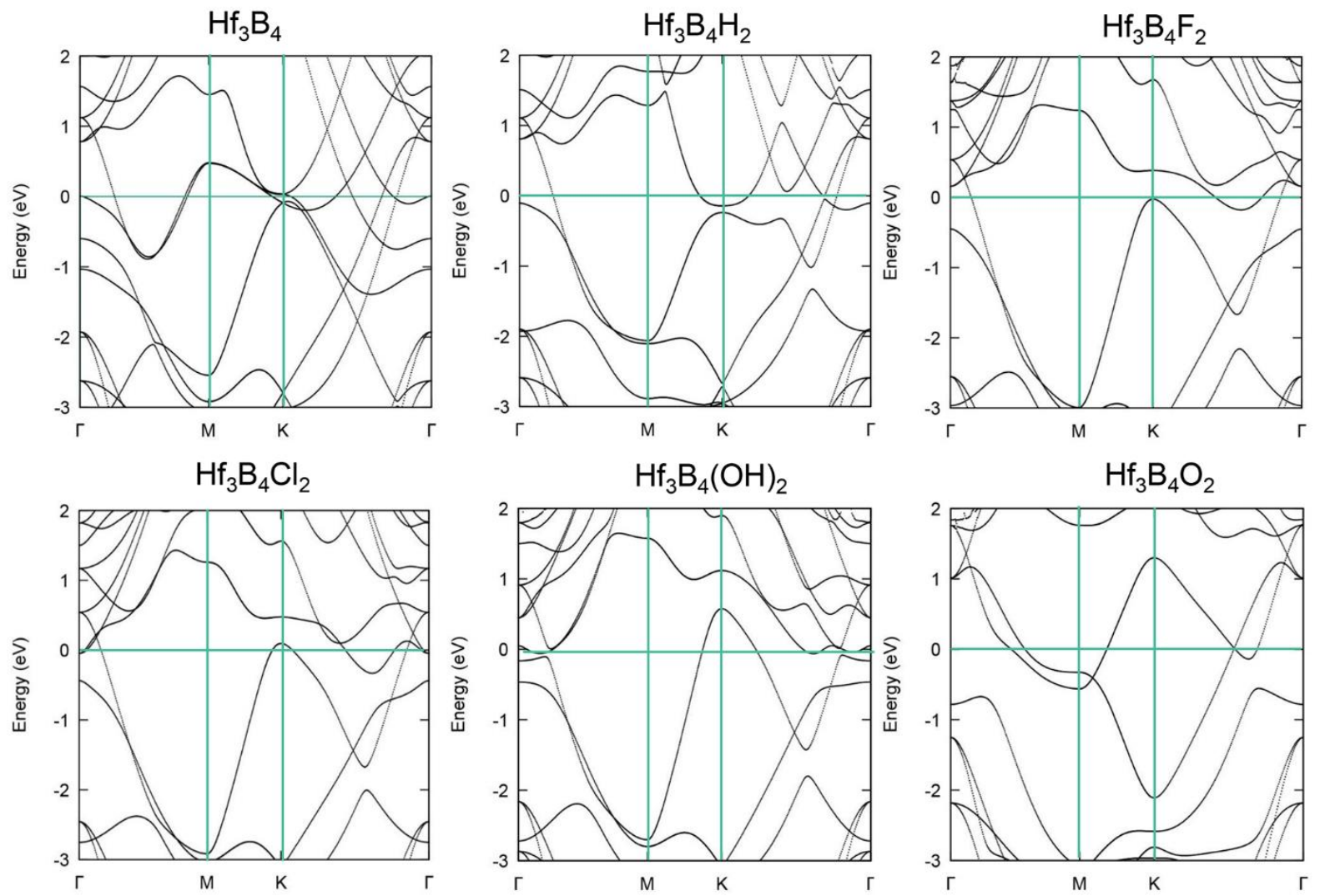

Figure S19. Calculated band structures of the $2 \mathrm{D} \mathrm{Hf}_{3} \mathrm{~B}_{4}$ with and without functional groups $T(T=\mathrm{H}$, $\mathrm{F}, \mathrm{Cl}, \mathrm{OH}$, and $\mathrm{O}$ ). 

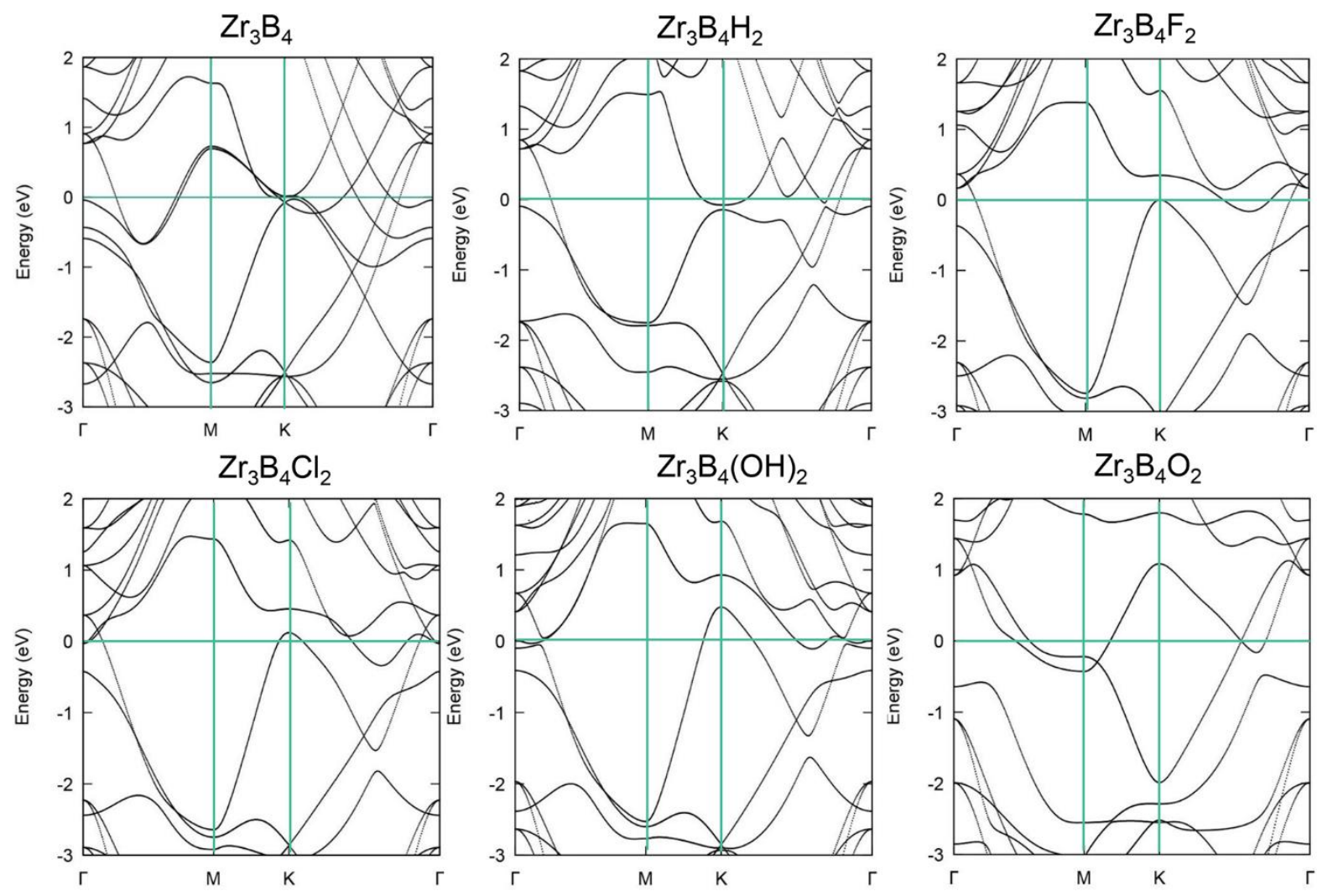

Figure S20. Calculated band structures of the $2 \mathrm{D} \mathrm{Zr}_{3} \mathrm{~B}_{4}$ with and without functional groups $T$ ( $T=\mathrm{H}$, $\mathrm{F}, \mathrm{Cl}, \mathrm{OH}$, and $\mathrm{O}$ ).

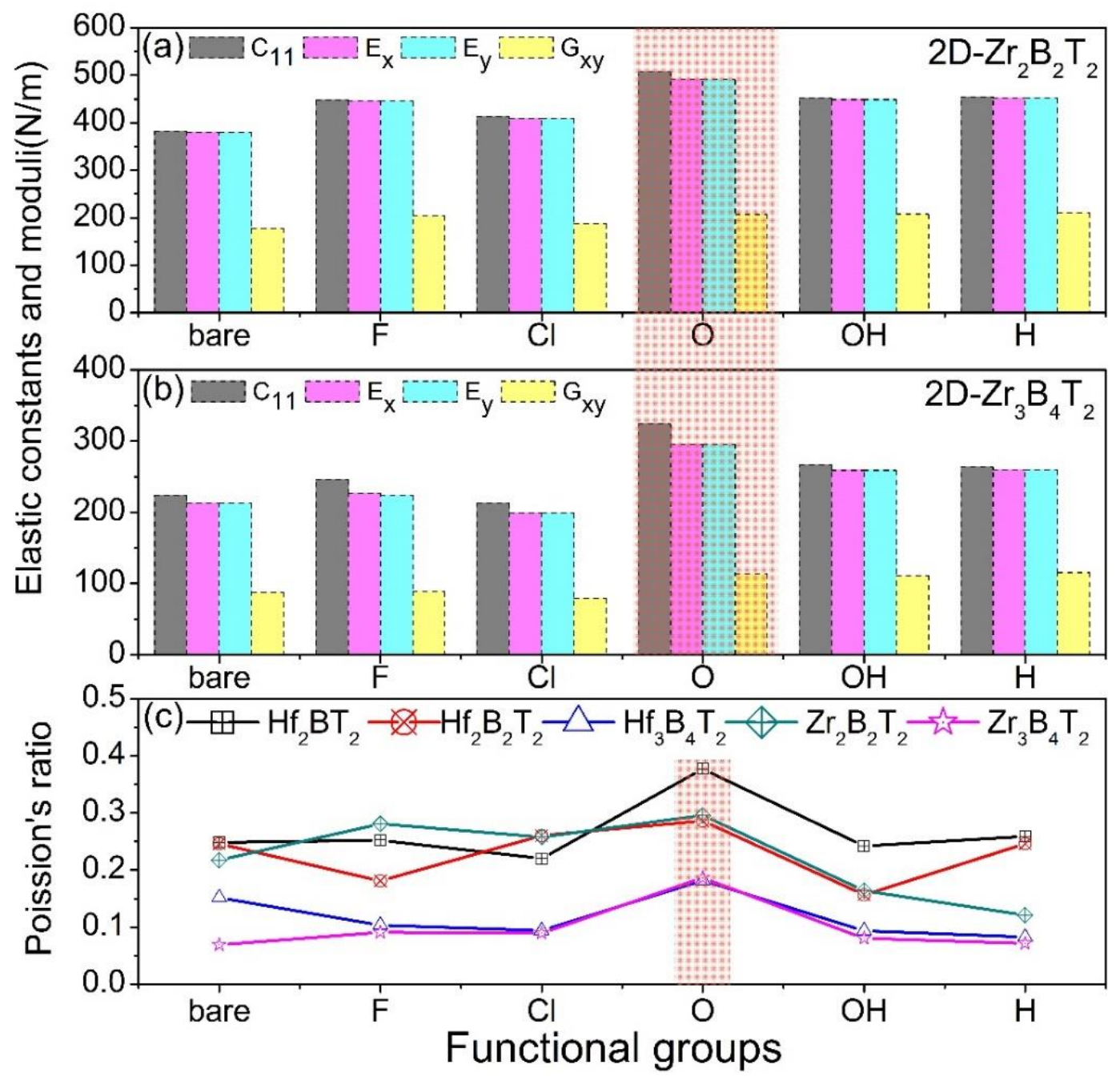

Figure S21. Calculated in-plane planar elastic constants $C_{\mathrm{ij}}$, Young's moduli $E_{\mathrm{x}}$ and $\mathrm{E}_{\mathrm{y}}$, Poisson's ratio $v$ and in-plane planar shear moduli $\mathrm{G}_{\mathrm{xy}}$ of boride MXenes. 
(a) $\mathrm{Hf}_{2} \mathrm{~B}$
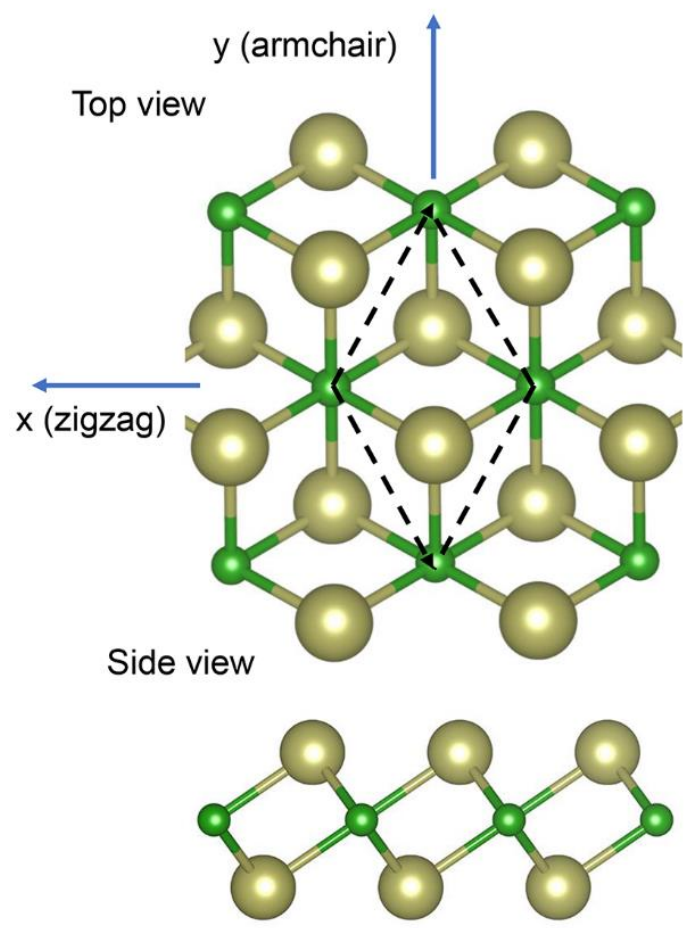

(b) $\mathrm{Hf}_{2} \mathrm{~B}_{2}$

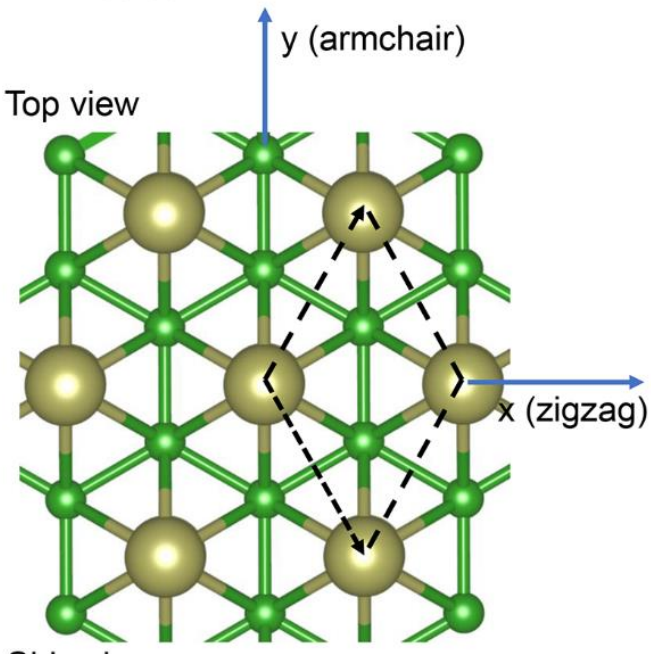

Side view

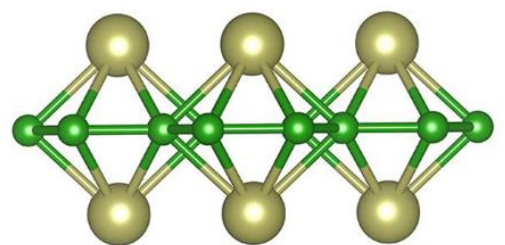

Figure S22. The schematic illustration of applied uniaxial and biaxial strain on (a) $2 \mathrm{D} \mathrm{Hf}_{2} \mathrm{~B}$ and (b) $2 \mathrm{D} \mathrm{Hf}_{2} \mathrm{~B}_{2}$.
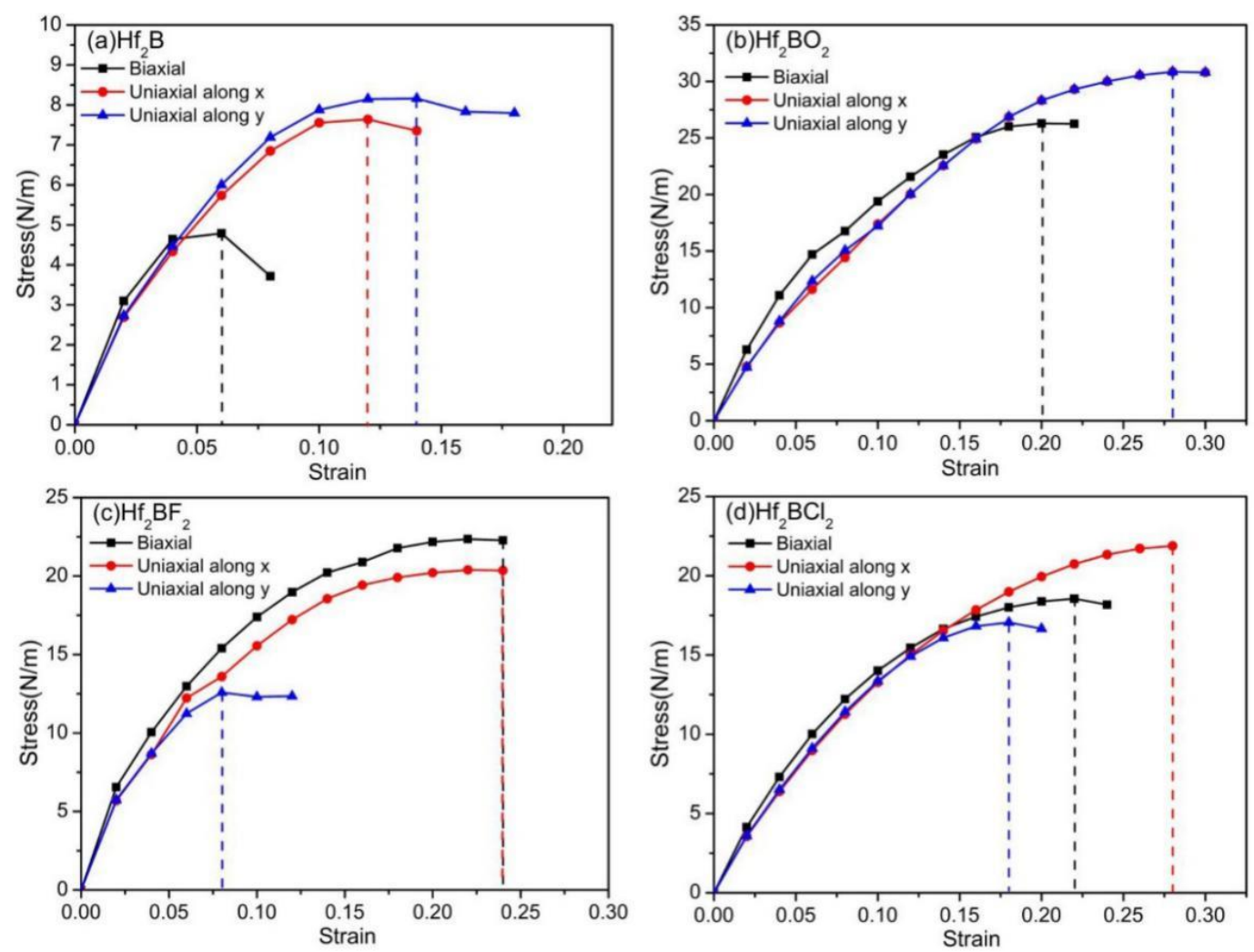

Figure S23. Calculated stress-strain curves of $2 \mathrm{D} \mathrm{Hf}_{2} \mathrm{~B}$ and $\mathrm{Hf}_{2} \mathrm{~B} T_{2}(T=\mathrm{O}, \mathrm{F}$, and $\mathrm{Cl})$ under biaxial and uniaxial tension. The maximum stress values are indicated by the vertical dash lines. 

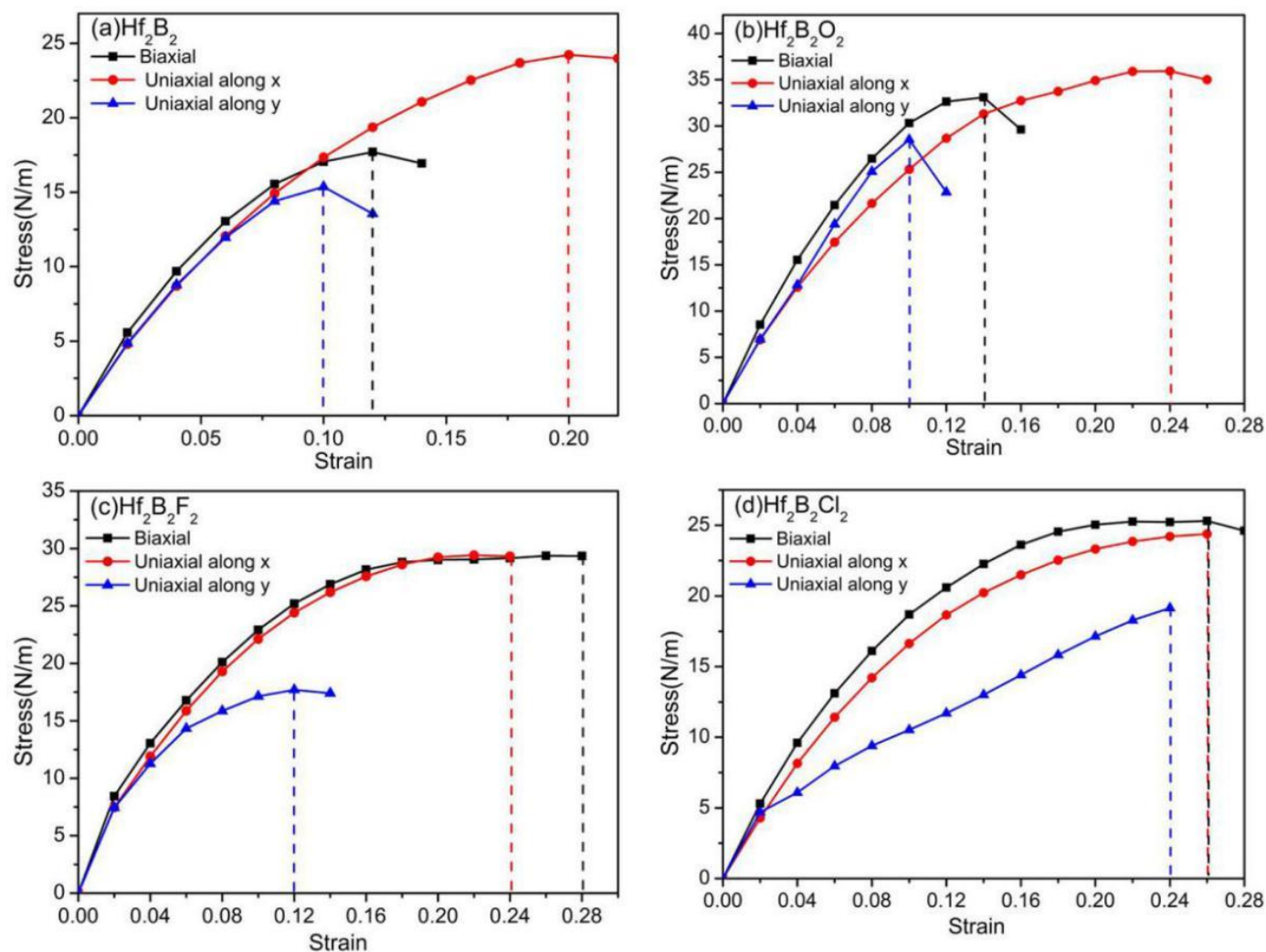

Figure S24. Calculated stress-strain curves of $2 \mathrm{D} \mathrm{Hf}_{2} \mathrm{~B}_{2}$ and $\mathrm{Hf}_{2} \mathrm{~B}_{2} T_{2}(T=\mathrm{O}, \mathrm{F}$, and $\mathrm{Cl})$ under biaxial and uniaxial tension. The maximum stress values are indicated by the vertical dash lines.
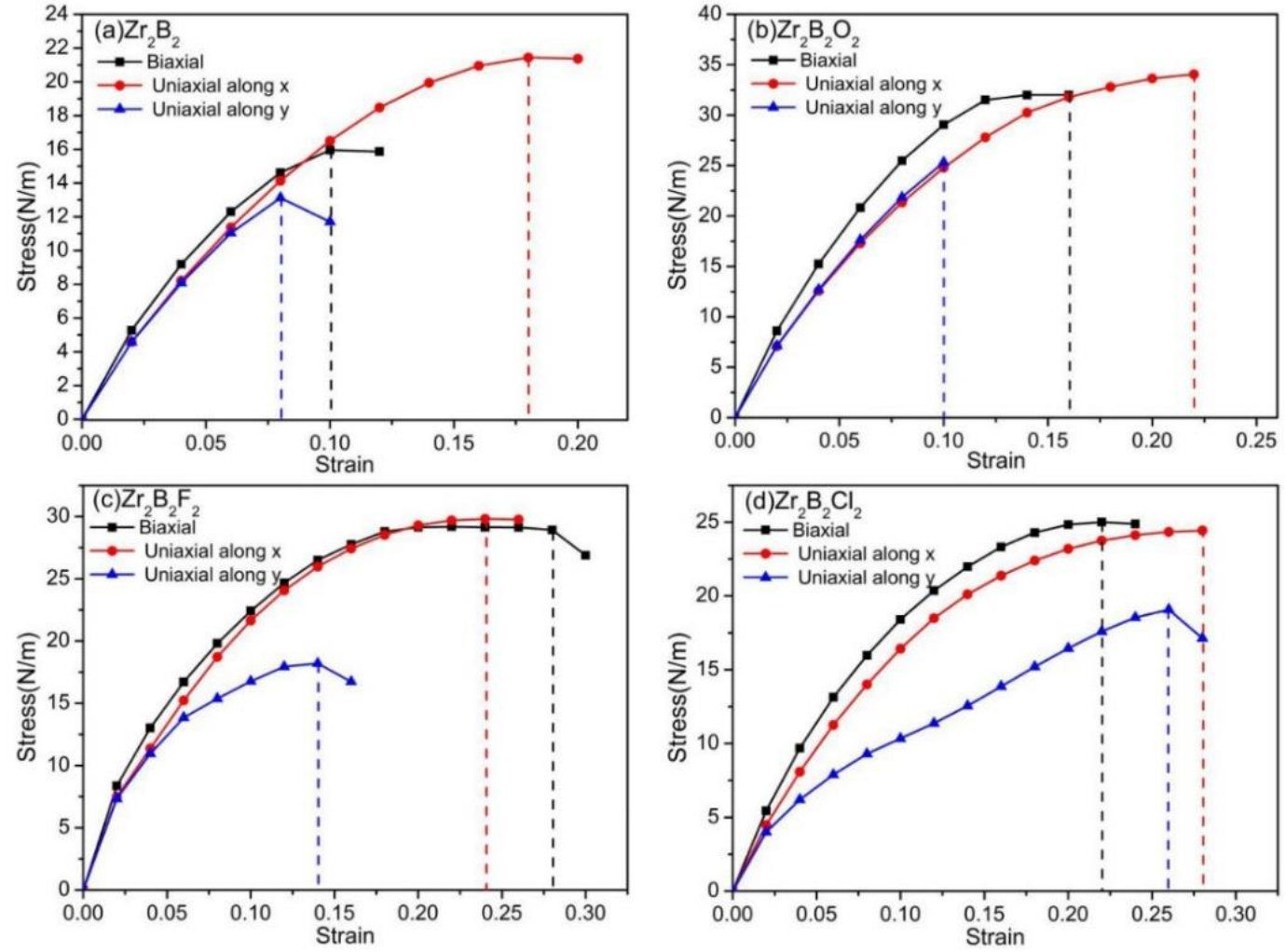

Figure S25. Calculated stress-strain curves of $2 \mathrm{D} \mathrm{Zr}_{2} \mathrm{~B}_{2}$ and $\mathrm{Zr}_{2} \mathrm{~B}_{2} T_{2}(T=\mathrm{O}, \mathrm{F}$, and $\mathrm{Cl})$ under biaxial and uniaxial tension. The maximum stress values are indicated by the vertical dash lines. 


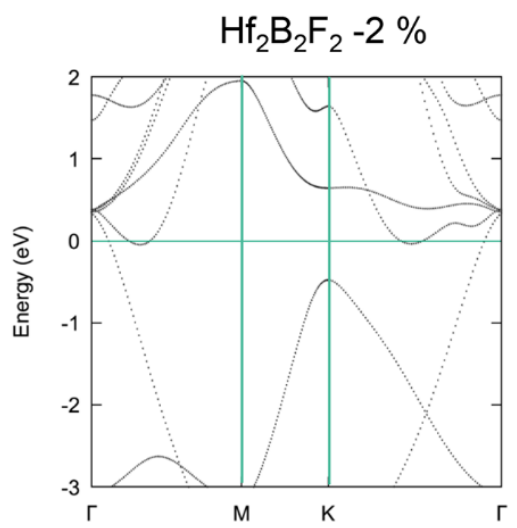

$\mathrm{Hf}_{2} \mathrm{~B}_{2} \mathrm{Cl}_{2}-2 \%$

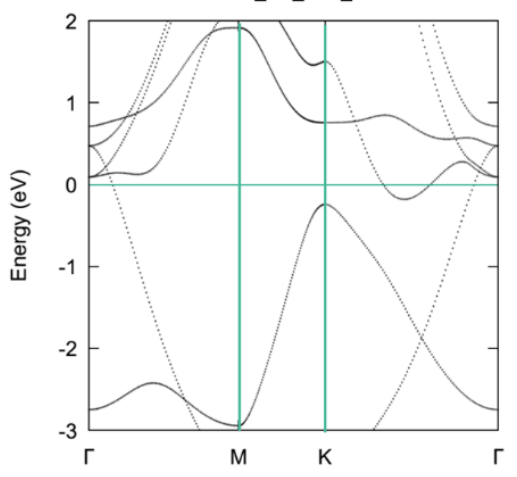

$\mathrm{Hf}_{2} \mathrm{~B}_{2} \mathrm{~F}_{2}-4 \%$

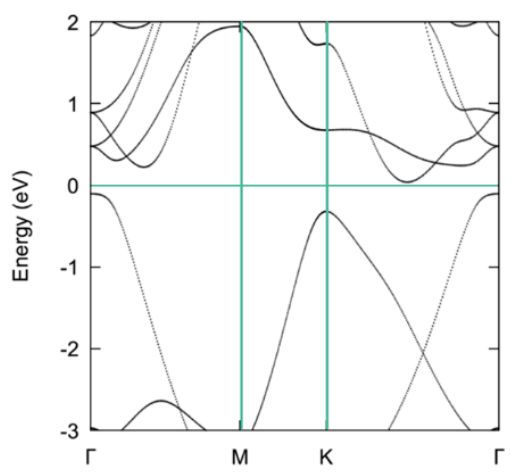

$\mathrm{Hf}_{2} \mathrm{~B}_{2} \mathrm{Cl}_{2}-4 \%$

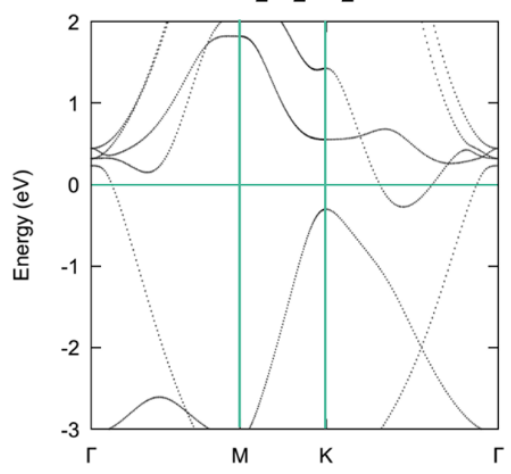

$\mathrm{Hf}_{2} \mathrm{~B}_{2} \mathrm{~F}_{2}-6 \%$

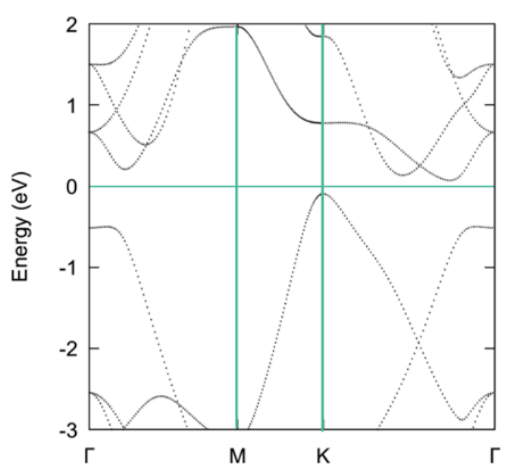

$\mathrm{Hf}_{2} \mathrm{~B}_{2} \mathrm{Cl}_{2}-6 \%$

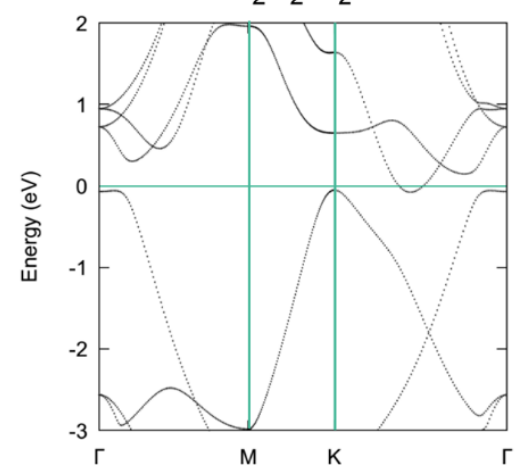

Figure S26. Calculated band structures of $2 \mathrm{D} \mathrm{Hf}_{2} \mathrm{~B}_{2} \mathrm{~F}_{2}$ and $\mathrm{Hf}_{2} \mathrm{~B}_{2} \mathrm{Cl}_{2}$ under compressive strains ranging from $2 \%$ to $6 \%$.
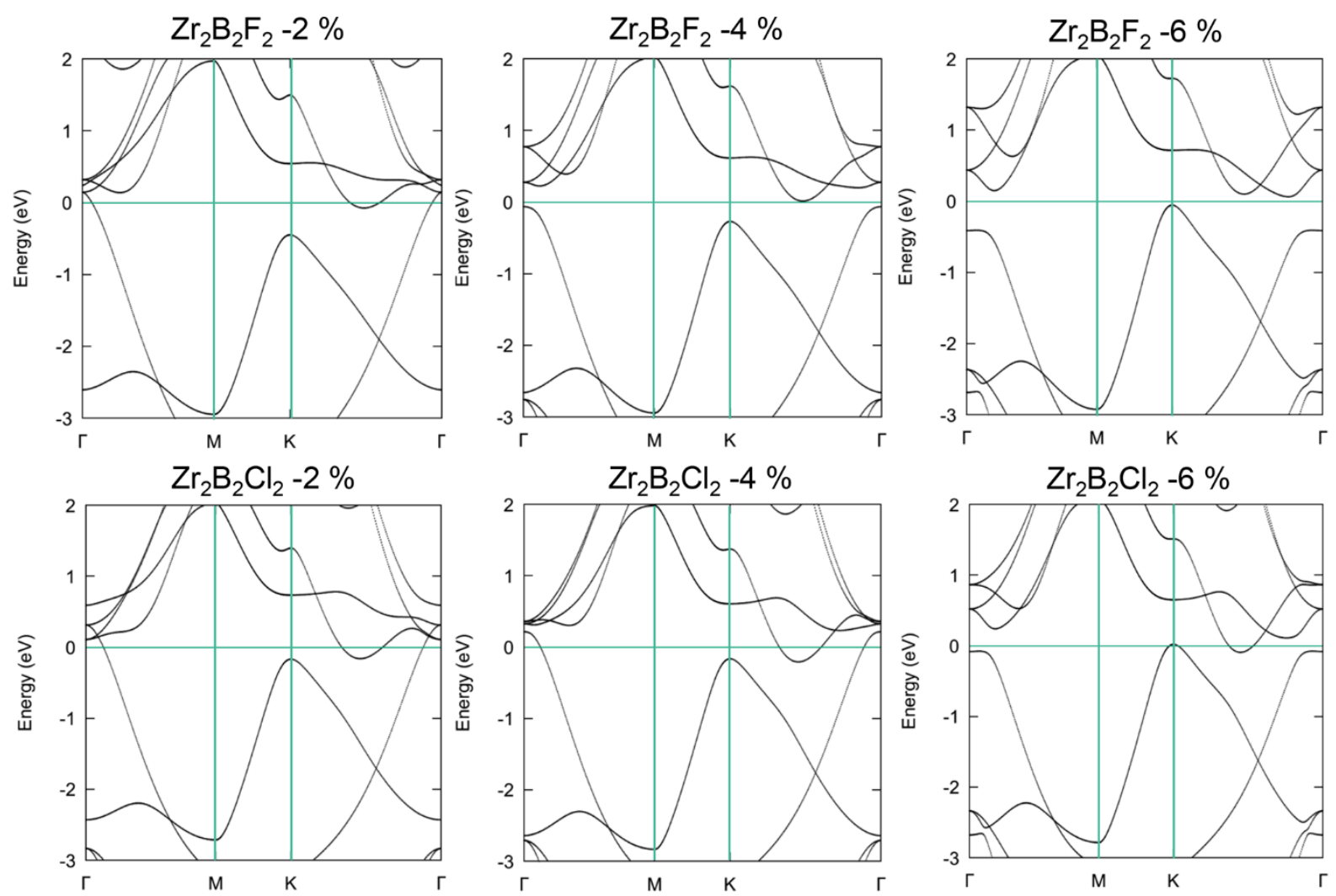

Figure S27. Calculated band structures of $2 \mathrm{D} \mathrm{Zr}_{2} \mathrm{~B}_{2} \mathrm{~F}_{2}$ and $\mathrm{Zr}_{2} \mathrm{~B}_{2} \mathrm{Cl}_{2}$ under compressive strains ranging from $2 \%$ to $6 \%$. 

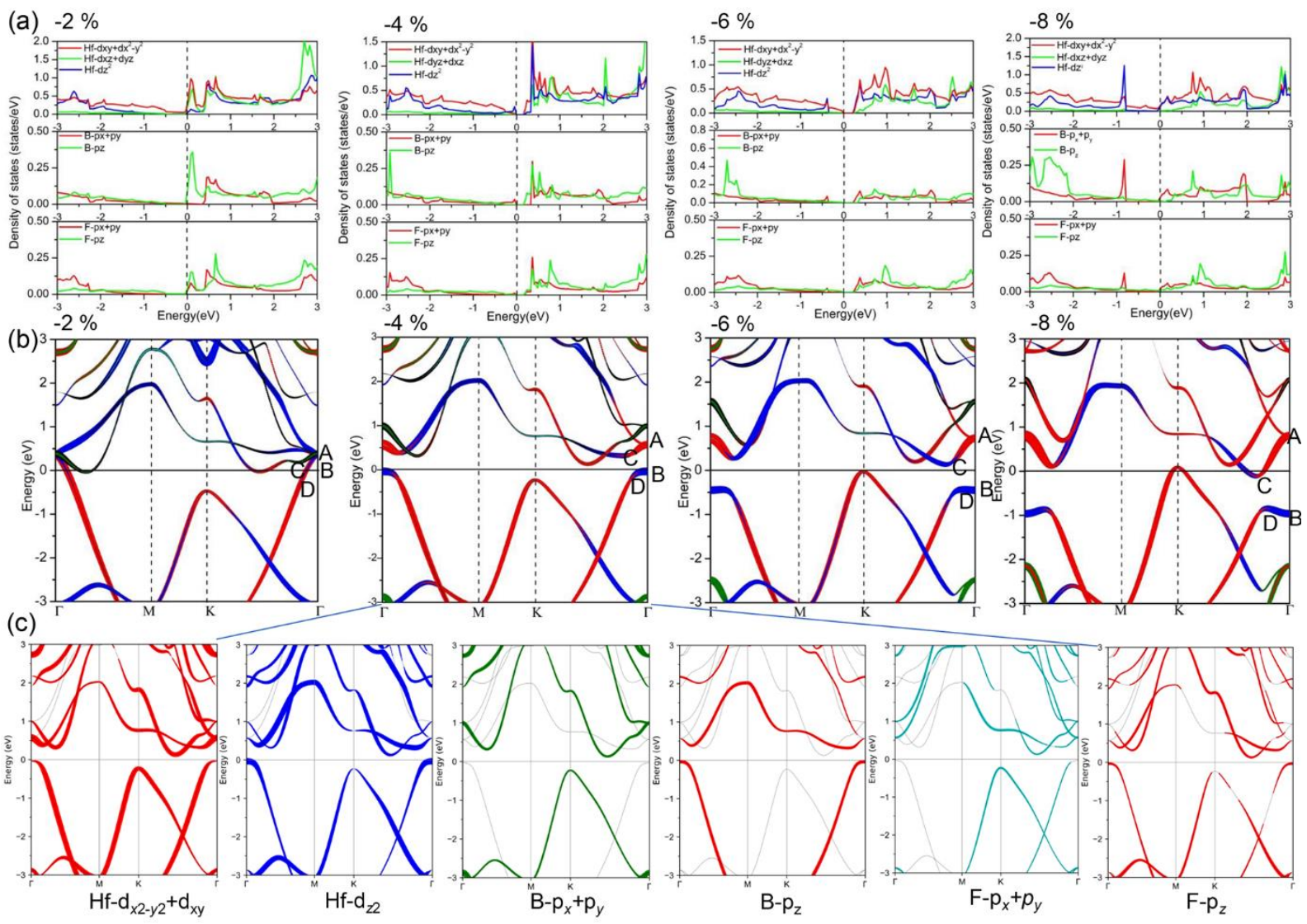

Figure S28. Calculated electronic structures of $2 \mathrm{D}-\mathrm{Hf}_{2} \mathrm{~B}_{2} \mathrm{~F}_{2}$ under $-2 \%,-4 \%,-6 \%$ and $-8 \%$ strains: (a) Partial density of states; (b) Orbital-resolved band structures (red, blue, green and absinthe-green

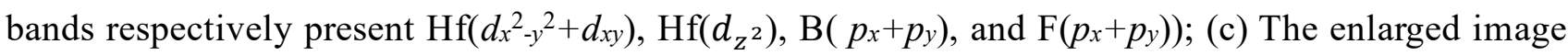
of orbital-resolved band structures under $-4 \%$ strain. 

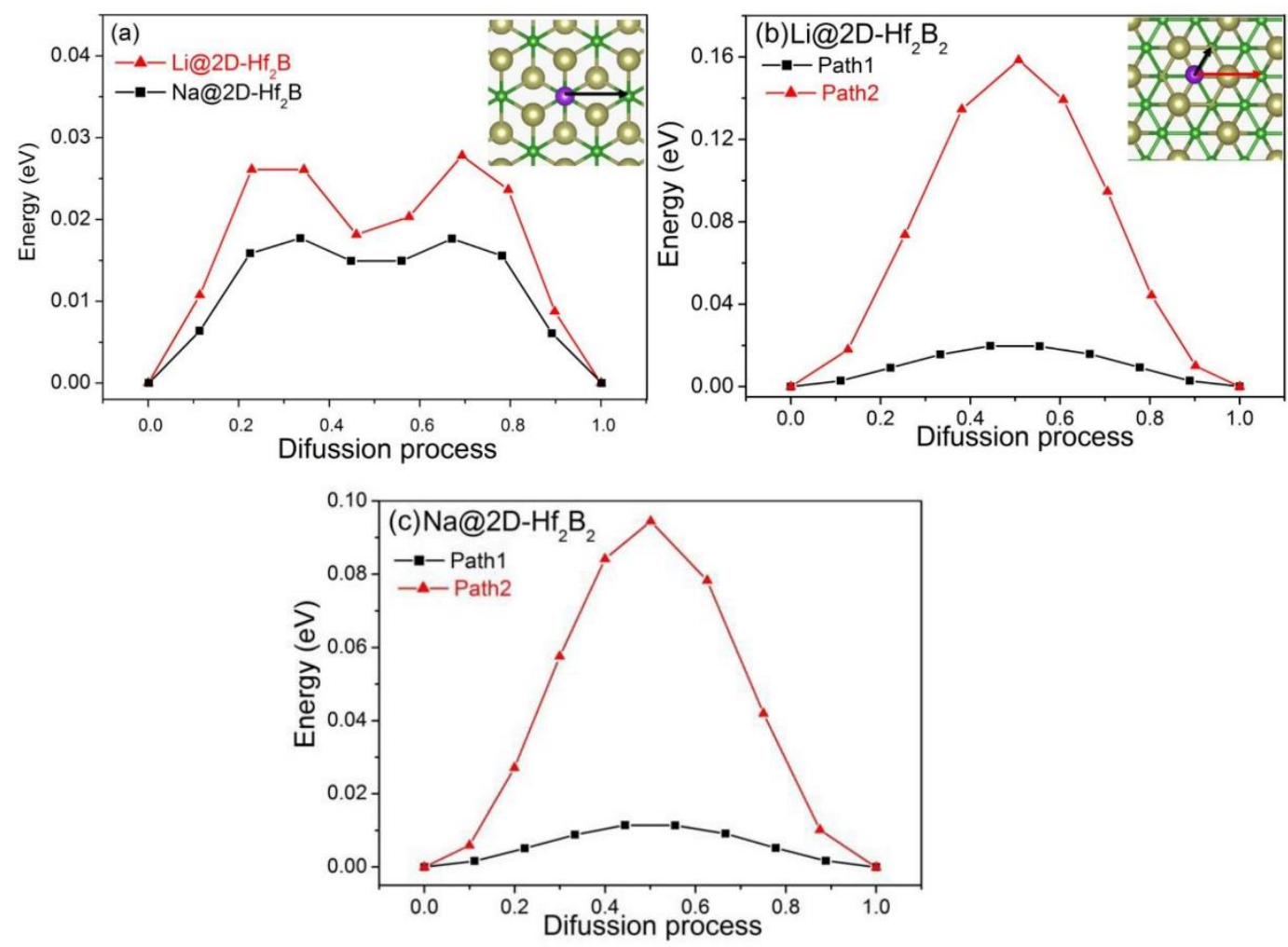

Figure S29. Considered adsorption sites and diffusion pathways for $\mathrm{Li}^{+}$and $\mathrm{Na}^{+}$on (a) $2 \mathrm{D} \mathrm{Hf} 2 \mathrm{~B}$, and (b-c) $2 \mathrm{D} \mathrm{Hf}_{2} \mathrm{~B}_{2}$. 


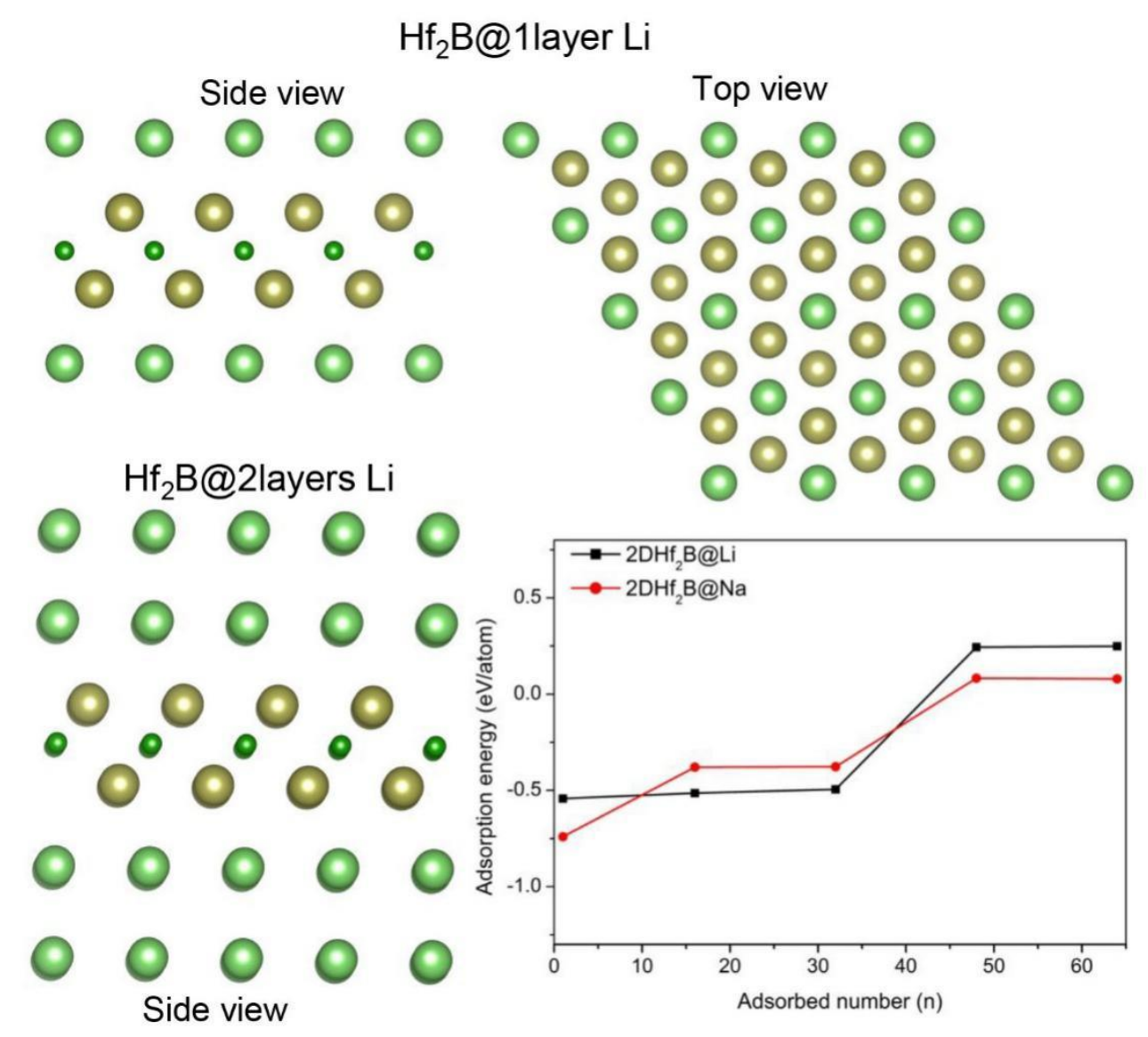

Figure S30. These most stable adsorption configurations and adsorption energies of $\mathrm{Li}^{+}$and $\mathrm{Na}^{+}$with various concentrations on $2 \mathrm{D} \mathrm{Hf}_{2} \mathrm{~B}$.

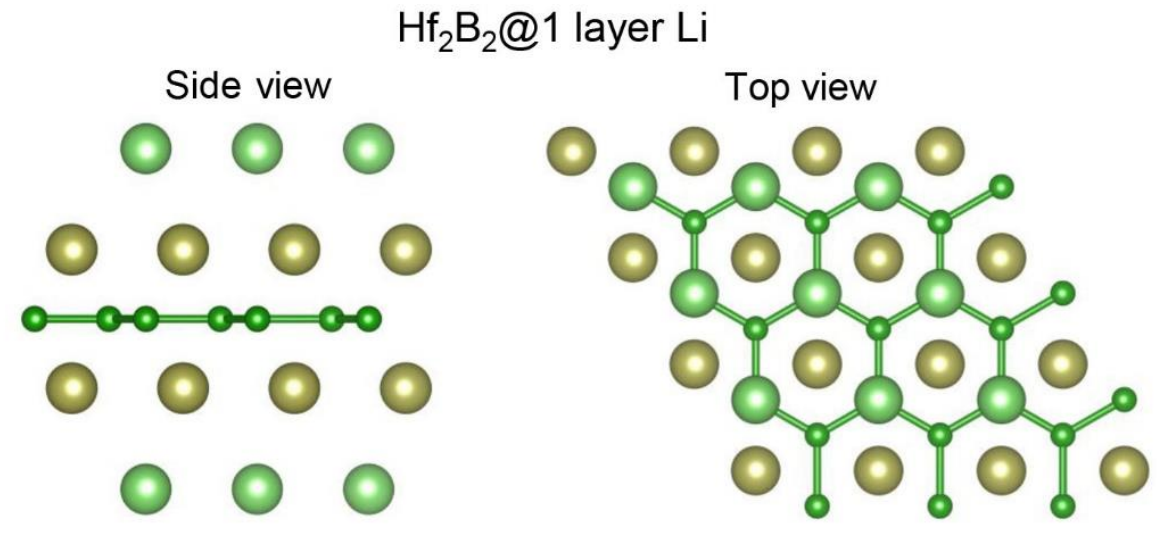

$\mathrm{Hf}_{2} \mathrm{~B}_{2} @ 2$ layers Li
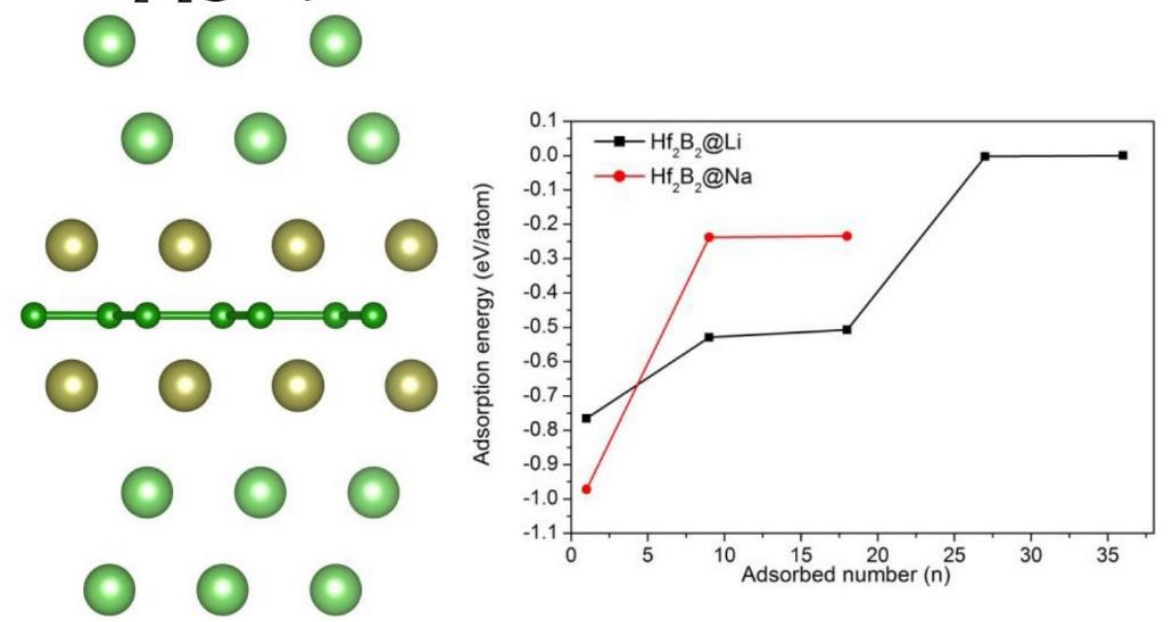

Side view 
Figure S31. These most stable adsorption configurations and adsorption energies of $\mathrm{Li}^{+}$and $\mathrm{Na}^{+}$with various concentrations on $2 \mathrm{D} \mathrm{Hf}_{2} \mathrm{~B}_{2}$.

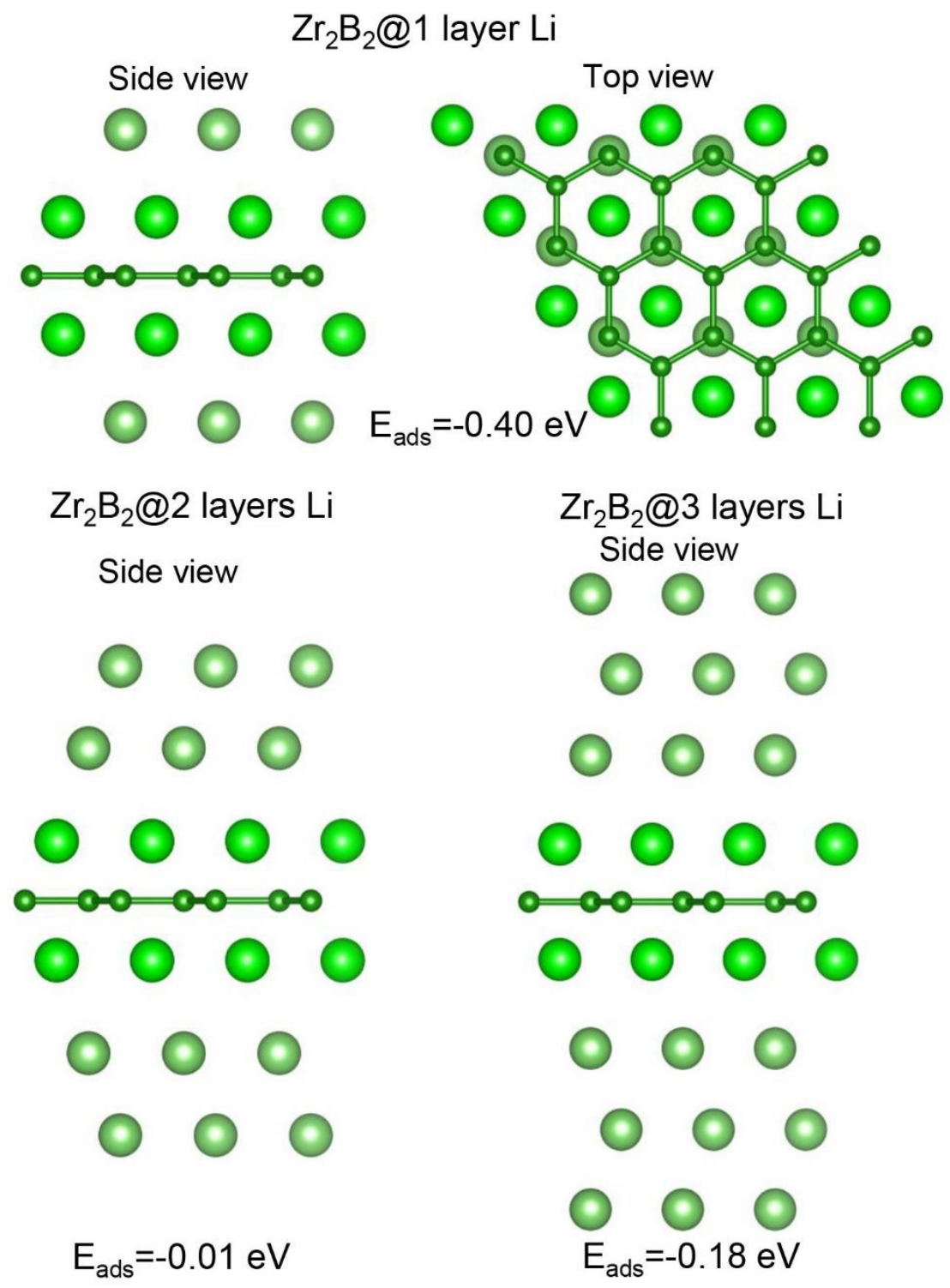

Figure S32. These most stable adsorption configurations and adsorption energies of $\mathrm{Li}^{+}$and $\mathrm{Na}^{+}$with various concentrations on $2 \mathrm{D} \mathrm{Zr}_{2} \mathrm{~B}_{2}$.

\section{Supporting Tables}

Table S1. The PAW pseudopotentials (version potpaw PBE.54) of all used elements.

\begin{tabular}{|c|c|c|c|c|c|c|c|}
\hline Element & $\begin{array}{c}\text { PAW } \\
\text { potential }\end{array}$ & Element & $\begin{array}{c}\text { PAW } \\
\text { potential }\end{array}$ & Element & $\begin{array}{c}\text { PAW } \\
\text { potential }\end{array}$ & Element & $\begin{array}{c}\text { PAW } \\
\text { potential }\end{array}$ \\
\hline $\mathrm{Ti}$ & $\mathrm{Ti} \_\mathrm{sv}$ & $\mathrm{Cd}$ & $\mathrm{Cd} \_\mathrm{sv}$ & $\mathrm{Si}$ & $\mathrm{Si}$ & $\mathrm{P}$ & $\mathrm{P}$ \\
\hline $\mathrm{Zr}$ & $\mathrm{Zr} \_\mathrm{sv}$ & $\mathrm{Al}$ & $\mathrm{Al}$ & $\mathrm{In}$ & $\mathrm{In} \_\mathrm{d}$ & $\mathrm{As}$ & $\mathrm{As}$ \\
\hline $\mathrm{Hf}$ & $\mathrm{Hf}$ _sv & $\mathrm{B}$ & $\mathrm{B}$ & $\mathrm{Tl}$ & $\mathrm{Tl} \_\mathrm{d}$ & $\mathrm{Sb}$ & $\mathrm{Sb}$ \\
\hline $\mathrm{Sn}$ & $\mathrm{Sn}$ & $\mathrm{Ga}$ & $\mathrm{Ga}$ & $\mathrm{Pb}$ & $\mathrm{Pb} \_\mathrm{d}$ & $\mathrm{Bi}$ & $\mathrm{Bi} \mathrm{d}$ \\
\hline
\end{tabular}


Table S2. Space group, atomic position, calculated energy (corrected with calculated zero-point energy at $0 \mathrm{~K}$ ) and lattice constants of $\mathrm{Hf}_{2} \mathrm{BiB}, \mathrm{Hf}_{2} \mathrm{PbB}, \mathrm{Hf}_{2} \mathrm{SnB}_{2}, \mathrm{Hf}_{2} \mathrm{InB}_{2}, \mathrm{Hf}_{3} \mathrm{~Pb}_{4}, \mathrm{Hf}_{3} \mathrm{SnB}_{4}, \mathrm{Hf}_{3} \mathrm{InB} 4$, $\mathrm{Zr}_{2} \mathrm{InB}_{2}, \mathrm{Zr}_{2} \mathrm{PbB}_{2}, \mathrm{Zr}_{2} \mathrm{TlB}_{2}, \mathrm{Zr}_{3} \mathrm{PbB}_{4}$, and $\mathrm{Zr}_{3} \mathrm{CdB}_{4}$.

\begin{tabular}{|c|c|c|c|c|c|}
\hline \multirow[t]{2}{*}{ Compound } & \multirow[t]{2}{*}{ Space group } & \multirow[t]{2}{*}{ Atomic position } & \multirow{2}{*}{$\begin{array}{l}\text { Energy } \\
\text { (eV/atom) }\end{array}$} & \multicolumn{2}{|c|}{ Lattice constants $(\AA)$} \\
\hline & & & & $\mathrm{a}$ & $\mathrm{c}$ \\
\hline \multirow[t]{3}{*}{$\mathrm{Hf}_{2} \mathrm{BiB}$} & $R 3 m$ & $\begin{array}{l}\text { Hf }(0.3333,0.6667,0.0496) \\
\text { Hf }(0.0000,0.0000,0.1612) \\
\text { B }(0.6667,0.3333,0.1044) \\
\operatorname{Bi}(0.6667,0.3333,0.2730)\end{array}$ & -9.49 & 3.497 & 22.076 \\
\hline & $P 6_{3} / m m c$ & $\begin{array}{l}\text { Hf }(0.3333,0.6667,0.0844) \\
\text { B }(0.0000,0.0000,0.0000) \\
\text { Bi }(0.6667,0.3333,0.2500)\end{array}$ & -9.46 & 3.523 & 14.489 \\
\hline & $P \overline{6} m 2$ & $\begin{array}{l}\text { Hf }(0.6667,0.3333,0.3320) \\
\operatorname{Hf}(0.3333,0.6667,0.1645) \\
\text { B }(0.0000,0.0000,0.2461) \\
\operatorname{Bi}(0.6667,0.3333,0.0000) \\
\operatorname{Bi}(0.0000,0.0000,0.5000)\end{array}$ & -9.46 & 3.503 & 14.700 \\
\hline \multirow[t]{3}{*}{$\mathrm{Hf}_{2} \mathrm{PbB}$} & $R 3 m$ & $\begin{array}{l}\text { Hf }(0.3333,0.6667,0.055) \\
\mathrm{Hf}(0.0000,0.0000,0.2783) \\
\mathrm{B}(0.3333,0.6667,0.6667) \\
\mathrm{Pb}(0.6667,0.3333,0.8333)\end{array}$ & -9.36 & 3.471 & 22.473 \\
\hline & $P 6_{3} / m m c$ & $\begin{array}{l}\mathrm{Hf}(0.3333,0.6667,0.0826) \\
\mathrm{B}(0.0000,0.0000,0.0000) \\
\mathrm{Pb}(0.6667,0.3333,0.2500)\end{array}$ & -9.37 & 3.468 & 14.967 \\
\hline & $P \overline{6} m 2$ & $\begin{array}{l}\mathrm{Hf}(0.3333,0.6667,0.3313) \\
\mathrm{Hf}(0.0000,0.0000,0.1669) \\
\mathrm{B}(0.6667,0.3333,0.2477) \\
\mathrm{Pb}(0.3333,0.6667,0.0000) \\
\mathrm{Pb}(0.6667,0.3333,0.5000)\end{array}$ & -9.36 & 3.458 & 15.098 \\
\hline $\mathrm{Hf}_{2} \operatorname{InB} \mathrm{B}_{2}$ & $P \overline{6} m 2$ & $\begin{array}{l}\text { Hf }(0.3333,0.6667,0.6989) \\
\text { B }(0.0000,0.0000,0.5000) \\
\text { B }(0.6667,0.3333,0.5000) \\
\text { In }(0.6667,0.3333,0.0000)\end{array}$ & -8.95 & 3.200 & 8.358 \\
\hline $\mathrm{Hf}_{2} \mathrm{SnB}_{2}$ & $P \overline{6} m 2$ & $\begin{array}{l}\text { Hf }(0.0000,0.0000,0.2969) \\
\text { B }(0.3333,0.6667,0.5000) \\
\text { B }(0.6667,0.3333,0.5000) \\
\text { Sn }(0.6667,0.3333,0.0000)\end{array}$ & -9.26 & 3.221 & 8.204 \\
\hline
\end{tabular}




\begin{tabular}{|c|c|c|c|c|c|}
\hline \multirow[t]{2}{*}{ Compound } & \multirow[t]{2}{*}{ Space group } & \multirow[t]{2}{*}{ Atomic position } & \multirow{2}{*}{$\begin{array}{l}\text { Energy } \\
\text { (eV/atom) }\end{array}$} & \multicolumn{2}{|c|}{ Lattice constants $(\AA)$} \\
\hline & & & & $\mathrm{a}$ & $\mathrm{c}$ \\
\hline $\mathrm{Hf}_{3} \operatorname{InB}_{4}$ & $P \overline{6} m 2$ & $\begin{array}{l}\text { Hf }(0.3333,0.6667,0.2875) \\
\text { Hf }(0.3333,0.6667,0.0000) \\
\text { B }(0.6667,0.3333,0.1467) \\
\text { B }(0.0000,0.0000,0.1474) \\
\text { In }(0.6667,0.3333,0.5000)\end{array}$ & -9.21 & 3.182 & 11.868 \\
\hline $\mathrm{Hf}_{3} \mathrm{~PB}_{4}$ & $P \overline{6} m 2$ & $\begin{array}{l}\text { Hf }(0.3333,0.6667,0.3270) \\
\text { Hf }(0.3333,0.6667,0.0000) \\
\text { B }(0.6667,0.3333,0.1637) \\
\text { B }(0.0000,0.0000,0.1667) \\
\text { P }(0.6667,0.3333,0.5000)\end{array}$ & -8.70 & 3.228 & 10.459 \\
\hline $\mathrm{Hf}_{3} \mathrm{SnB}_{4}$ & $P \overline{6} m 2$ & $\begin{array}{l}\text { Hf }(0.3333,0.6667,0.2915) \\
\text { Hf }(0.3333,0.6667,0.000) \\
\text { B }(0.6667,0.3333,0.1478) \\
\text { B }(0.0000,0.0000,0.1483) \\
\text { Sn }(0.6667,0.3333,0.5000)\end{array}$ & -9.40 & 3.179 & 11.787 \\
\hline $\mathrm{Zr}_{2} \operatorname{InB}_{2}$ & $P \overline{6} m 2$ & $\begin{array}{l}\text { Hf }(0.6667,0.3333,0.6980) \\
\text { B }(0.3333,0.6667,0.0000) \\
\text { B }(0.0000,0.0000,0.5000) \\
\text { In }(0.3333,0.6667,0.5000)\end{array}$ & -8.00 & 3.233 & 8.485 \\
\hline $\mathrm{Zr}_{2} \mathrm{PbB}_{2}$ & $P \overline{6} m 2$ & $\begin{array}{l}\mathrm{Zr}(0.0000,0.0000,0.6976) \\
\mathrm{Pb}(0.3333,0.6667,0.0000) \\
\mathrm{B}(0.6667,0.3333,0.5000) \\
\mathrm{B}(0.3333,0.6667,0.5000)\end{array}$ & -8.15 & 3.276 & 8.415 \\
\hline $\mathrm{Zr}_{2} \mathrm{TlB}_{2}$ & $P \overline{6} m 2$ & $\begin{array}{l}\mathrm{Zr}(0.3333,0.6667,0.6967) \\
\mathrm{Tl}(0.6667,0.3333,0.0000) \\
\mathrm{B}(0.0000,0.0000,0.5000) \\
\mathrm{B}(0.6667,0.3333,0.5000)\end{array}$ & -7.85 & 3.254 & 8.486 \\
\hline $\mathrm{Zr}_{3} \mathrm{PbB}_{4}$ & $P \overline{6} \mathrm{~m} 2$ & $\begin{array}{l}\mathrm{Zr}(0.6667,0.3333,0.2871) \\
\mathrm{Zr}(0.6667,0.3333,0.0000) \\
\mathrm{B}(0.3333,0.6667,0.1465) \\
\mathrm{B}(0.0000,0.0000,0.1467) \\
\mathrm{Pb}(0.3333,0.6667,0.5000)\end{array}$ & -8.40 & 3.226 & 12.035 \\
\hline $\mathrm{Zr}_{3} \mathrm{CdB}_{4}$ & $P \overline{6} m 2$ & $\begin{array}{l}\mathrm{Zr}(0.3333,0.6667,0.2850) \\
\mathrm{Zr}(0.3333,0.6667,0.0000) \\
\mathrm{B}(0.6667,0.3333,0.1452) \\
\mathrm{B}(0.0000,0.0000,0.1456) \\
\mathrm{Cd}(0.6667,0.3333,0.5000)\end{array}$ & -8.02 & 3.185 & 12.197 \\
\hline
\end{tabular}


Table S3. Calculated enthalpies above the convex hull (meV/atom) of all predicted structures.

\begin{tabular}{lccccccccc}
\hline & \multicolumn{3}{c}{$\mathrm{Ti}-\mathrm{A}-\mathrm{B}$} & \multicolumn{3}{c}{$\mathrm{Zr}-\mathrm{A}-\mathrm{B}$} & \multicolumn{3}{c}{$\mathrm{Hf}-\mathrm{A}-\mathrm{B}$} \\
\hline $\mathrm{Al}$ & 232 & 10 & 7 & 257 & 151 & 45 & 227 & 28 & 24 \\
$\mathrm{Si}$ & 424 & 79 & 51 & 388 & 92 & 108 & 386 & 89 & 164 \\
$\mathrm{P}$ & 297 & 90 & 66 & 155 & 147 & 105 & 76 & 47 & 0 \\
$\mathrm{Ga}$ & 59 & 57 & 7 & 260 & 55 & 41 & 205 & 25 & 22 \\
$\mathrm{Ge}$ & 226 & 14 & 7 & 340 & 80 & 50 & 228 & 31 & 30 \\
$\mathrm{As}$ & 243 & 98 & 66 & 170 & 141 & 99 & 117 & 132 & 97 \\
$\mathrm{Sb}$ & 206 & 260 & 89 & 116 & 105 & 71 & 46 & 127 & 83 \\
$\mathrm{Bi}$ & 111 & 133 & 119 & 18 & 89 & 74 & 0 & 139 & 104 \\
$\mathrm{~Pb}$ & 82 & 39 & 38 & 42 & 0 & 4 & 0 & 36 & 34 \\
$\mathrm{Sn}$ & 147 & 2 & 3 & 159 & 8 & 9 & 47 & 0 & 3 \\
$\mathrm{In}$ & 68 & 0 & 4 & 62 & 0 & 5 & 46 & 0 & 6 \\
$\mathrm{Cd}$ & 173 & 40 & 32 & 113 & 5 & 0 & 37 & 24 & 14 \\
$\mathrm{Tl}$ & 150 & 34 & 33 & 39 & 0 & 6 & 14 & 9 & 12 \\
\hline
\end{tabular}


Table S4. Calculated Bader and Mulliken charges, integrated crystal orbital Hamilton population (ICOHP), $\mathrm{k}$ represents the force constant of each chemical bonds and $\mathrm{F}_{\mathrm{C}}$ summation of force constants of springs connected to a $\mathrm{M}, \mathrm{A}$, or $\mathrm{X}(\mathrm{B})$ atom of the predicted MAX phases, especially for the ICOHP only include $\mathrm{M}_{2} \mathrm{BiB}, \mathrm{M}_{2} \mathrm{PbB}$ and $\mathrm{M}_{2} \mathrm{InB}_{2}(\mathrm{M}=\mathrm{Ti}, \mathrm{Zr}$, and $\mathrm{Hf})$, predicted $2 \mathrm{D}$ borides and competing binary phases, and the symbol '-' represents the negligible bonds.

\begin{tabular}{|c|c|c|c|c|c|c|c|c|c|c|c|c|c|c|c|}
\hline \multirow[b]{2}{*}{ MAX } & \multicolumn{3}{|c|}{ Bader charge $(|e|)$} & \multicolumn{3}{|c|}{ Mulliken charge $(|e|)$} & \multicolumn{3}{|c|}{$-\mathrm{ICOHP}(\mathrm{eV})$} & \multicolumn{3}{|c|}{$\mathrm{k}(\mathrm{eV} / \AA)$} & \multicolumn{3}{|c|}{$\mathrm{FC}(\mathrm{eV} / \AA)$} \\
\hline & M & A & B & M & A & B & $\mathrm{M}-\mathrm{X}$ & M-A & $X-X$ & M-B & M-A & $\mathrm{X}-\mathrm{X}$ & $\mathrm{FC}(\mathrm{M})$ & $\mathrm{FC}(\mathrm{A})$ & $\mathrm{FC}(\mathrm{X})$ \\
\hline $\mathrm{Ti}_{2} \mathrm{BiB}$ & 1.27 & -0.69 & -1.85 & 0.51 & -0.22 & -0.75 & 3.15 & 1.37 & 0.28 & 4.99 & 1.12 & 0.21 & 40.13 & 17.80 & 29.30 \\
\hline $\mathrm{Zr}_{2} \mathrm{BiB}$ & 1.39 & -0.80 & -1.97 & 0.52 & -0.27 & -0.75 & 3.44 & 1.69 & 0.35 & 4.44 & 1.27 & 0.42 & 43.21 & 18.35 & 25.59 \\
\hline $\mathrm{Hf}_{2} \mathrm{BiB}$ & 1.41 & -0.79 & -2.03 & 0.86 & -0.47 & -1.27 & 3.45 & 1.74 & 0.52 & 5.08 & 1.31 & 0.51 & 44.44 & 15.97 & 28.96 \\
\hline $\mathrm{Ti}_{2} \mathrm{PbB}$ & 1.29 & -0.69 & -1.89 & 0.52 & -0.37 & -0.67 & 3.31 & 1.29 & 0.39 & 5.42 & 1.30 & 0.42 & 41.51 & 15.90 & 31.27 \\
\hline $\mathrm{Zr}_{2} \mathrm{PbB}$ & 1.40 & -0.78 & -2.02 & 0.56 & -0.41 & -0.73 & 3.57 & 1.54 & 0.50 & 4.78 & 1.31 & 0.49 & 42.32 & 15.97 & 27.00 \\
\hline $\mathrm{Hf}_{2} \mathrm{PbB}$ & 1.42 & -0.79 & -2.05 & 0.98 & -0.67 & -1.29 & 3.58 & 1.61 & 0.75 & 5.45 & 1.37 & 0.71 & 45.51 & 18.06 & 32.72 \\
\hline $\mathrm{Ti}_{2} \operatorname{InB}_{2}$ & 1.19 & -0.66 & -0.86 & 0.53 & -0.25 & -0.41 & 1.60 & 1.53 & 5.95 & 3.32 & 1.81 & 7.67 & 35.98 & 25.23 & 42.98 \\
\hline $\mathrm{Zr}_{2} \operatorname{InB}_{2}$ & 1.24 & -0.72 & -0.89 & 0.54 & -0.27 & -0.41 & 1.78 & 1.73 & 5.32 & 3.32 & 1.85 & 5.03 & 45.98 & 18.52 & 35.03 \\
\hline $\mathrm{Hf}_{2} \operatorname{InB}{ }_{2}$ & 1.25 & -0.70 & -0.90 & 1.00 & -0.66 & -0.67 & 1.78 & 1.77 & 5.76 & 3.51 & 2.01 & 5.59 & 47.58 & 21.79 & 37.87 \\
\hline $2 \mathrm{D}-\mathrm{Hf}_{2} \mathrm{~B}$ & 0.89 & - & -1.98 & 0.68 & - & -1.36 & 3.02 & - & - & - & - & - & - & - & - \\
\hline $2 \mathrm{D}-\mathrm{Hf}_{2} \mathrm{~B}_{2}$ & 0.86 & - & -0.86 & 0.62 & - & -0.62 & 1.58 & - & 5.28 & - & - & 5.48 & - & - & - \\
\hline $2 \mathrm{D}-\mathrm{Ti}_{2} \mathrm{~B}_{2}$ & 0.84 & - & -0.84 & 0.46 & - & -0.46 & 1.51 & - & 5.15 & - & - & 5.25 & - & - & - \\
\hline $2 \mathrm{D}-\mathrm{Zr}_{2} \mathrm{~B}_{2}$ & 0.85 & - & -0.85 & 0.47 & - & -0.47 & 1.65 & - & 5.07 & - & - & 5.50 & - & - & - \\
\hline $\mathrm{B}_{36}$ & - & - & - & - & - & - & - & - & 4.99 & - & - & 4.54 & - & - & - \\
\hline $\mathrm{TiB}_{2}$ & 1.40 & - & -0.70 & 1.44 & - & -0.72 & 1.26 & - & 7.04 & 1.12 & - & 6.01 & - & - & - \\
\hline $\mathrm{ZrB}_{2}$ & 1.50 & - & -0.75 & 1.46 & - & 0.73 & 1.75 & - & 5.97 & 1.32 & - & 5.98 & - & - & - \\
\hline $\mathrm{HfB}_{2}$ & 1.52 & - & -0.76 & 1.46 & - & -0.73 & 1.74 & - & 5.95 & 1.32 & & 5.98 & - & - & - \\
\hline $\mathrm{Hf}_{2} \mathrm{Bi}$ & 0.51 & -1.02 & - & 0.35 & -0.69 & - & - & 1.51 & - & - & 1.40 & - & - & - & - \\
\hline $\mathrm{Hf}_{3} \mathrm{~Pb}$ & 0.41 & -1.22 & - & 0.41 & -1.30 & - & - & 1.24 & - & - & 1.12 & - & - & - & - \\
\hline $\mathrm{Ti}_{3} \mathrm{In}$ & 0.42 & -1.27 & - & 0.67 & -2.00 & - & - & 1.19 & - & - & 1.01 & - & - & - & - \\
\hline $\mathrm{Hf}_{2} \mathrm{In}_{5}$ & 0.84 & -0.32 & - & 1.02 & -0.39 & - & - & 1.38 & - & - & 1.21 & - & - & - & - \\
\hline $\mathrm{Zr}_{3} \mathrm{In}$ & 0.4 & -1.2 & - & 0.12 & -0.36 & - & - & 1.68 & - & - & 1.45 & - & - & - & \\
\hline $\mathrm{Ti}_{2} \mathrm{AlC}^{1}$ & - & - & - & - & - & - & 3.86 & 2.01 & - & 7.11 & 1.74 & 0.44 & 46.71 & 15.07 & 51.52 \\
\hline $\mathrm{Ti}_{3} \mathrm{AlC}_{2}{ }^{1}$ & - & - & - & - & - & - & 4.23 & 2.02 & - & 8.31 & 1.76 & 0.52 & 49.17 & 14.70 & 49.51 \\
\hline
\end{tabular}


Table S5. The adsorption energies (eV/atom) of functional group $\mathrm{T}(\mathrm{T}=\mathrm{F}, \mathrm{Cl}, \mathrm{H}, \mathrm{O}, \mathrm{OH}, \mathrm{Li}$, and $\mathrm{Na}$ ) on $2 \mathrm{D} \mathrm{Hf}_{2} \mathrm{~B}, \mathrm{Hf}_{2} \mathrm{~B}_{2}, \mathrm{Zr}_{2} \mathrm{~B}_{2}, \mathrm{Hf}_{3} \mathrm{~B}_{4}$, and $\mathrm{Zr}_{3} \mathrm{~B}_{4}$ at different adsorption sites. The symbol '-' represents unstable adsorption site.

\begin{tabular}{|c|c|c|c|c|}
\hline & -top Hf & Hollow A & Hollow B & HollowA, B \\
\hline $\mathrm{Hf}_{2} \mathrm{BO}_{2}$ & - & -6.10 & -5.38 & -5.61 \\
\hline $\mathrm{Hf}_{2} \mathrm{BH}_{2}$ & - & -1.31 & -0.94 & -1.15 \\
\hline $\mathrm{Hf}_{2} \mathrm{BF}_{2}$ & -4.90 & -5.66 & -5.25 & -4.27 \\
\hline $\mathrm{Hf}_{2} \mathrm{~B}(\mathrm{OH})_{2}$ & -7.41 & -7.95 & -7.63 & -7.80 \\
\hline $\mathrm{Hf}_{2} \mathrm{BCl}_{2}$ & -3.37 & -4.41 & -4.10 & -4.26 \\
\hline $\mathrm{Hf}_{2} \mathrm{BLi}_{2}$ & -0.43 & -0.47 & -0.43 & -0.35 \\
\hline $\mathrm{Hf}_{2} \mathrm{BNa}_{2}$ & -0.26 & -0.27 & -0.26 & -0.24 \\
\hline $\mathrm{Hf}_{2} \mathrm{~B}_{2} \mathrm{O}_{2}$ & - & -5.78 & -5.76 & -5.79 \\
\hline $\mathrm{Hf}_{2} \mathrm{~B}_{2} \mathrm{~F}_{2}$ & -5.35 & -5.38 & -5.36 & -5.37 \\
\hline $\mathrm{Hf}_{2} \mathrm{~B}_{2} \mathrm{Cl}_{2}$ & -3.93 & -3.95 & -3.84 & -3.93 \\
\hline $\mathrm{Hf}_{2} \mathrm{~B}_{2}(\mathrm{OH})_{2}$ & -7.76 & -7.80 & -7.75 & -7.77 \\
\hline $\mathrm{Hf}_{2} \mathrm{~B}_{2} \mathrm{H}_{2}$ & -1.25 & -1.28 & -1.26 & -1.27 \\
\hline $\mathrm{Hf}_{2} \mathrm{~B}_{2} \mathrm{Li}_{2}$ & -0.28 & -0.50 & -0.44 & - \\
\hline $\mathrm{Hf}_{2} \mathrm{~B}_{2} \mathrm{Na}_{2}$ & -0.08 & -0.18 & -0.17 & - \\
\hline $\mathrm{Zr}_{2} \mathrm{~B}_{2} \mathrm{O}_{2}$ & - & -5.65 & -5.63 & -5.67 \\
\hline $\mathrm{Zr}_{2} \mathrm{~B}_{2} \mathrm{~F}_{2}$ & -5.51 & -5.54 & -5.53 & -5.52 \\
\hline $\mathrm{Zr}_{2} \mathrm{~B}_{2} \mathrm{Cl}_{2}$ & - & -4.08 & -4.07 & -4.06 \\
\hline $\mathrm{Zr}_{2} \mathrm{~B}_{2} \mathrm{H}_{2}$ & - & -1.28 & -1.26 & -1.27 \\
\hline $\mathrm{Zr}_{2} \mathrm{~B}_{2}(\mathrm{OH})_{2}$ & - & -7.88 & -7.86 & -7.87 \\
\hline $\mathrm{Zr}_{2} \mathrm{~B}_{2} \mathrm{Li}_{2}$ & -0.57 & -0.69 & -0.38 & - \\
\hline $\mathrm{Zr}_{2} \mathrm{~B}_{2} \mathrm{Na}_{2}$ & -0.25 & -0.53 & -0.26 & - \\
\hline $\mathrm{Hf}_{3} \mathrm{~B}_{4} \mathrm{O}_{2}$ & - & -7.96 & -7.90 & -8.00 \\
\hline $\mathrm{Hf}_{3} \mathrm{~B}_{4} \mathrm{~F}_{2}$ & - & -6.23 & -5.72 & -6.15 \\
\hline $\mathrm{Hf}_{3} \mathrm{~B}_{4} \mathrm{Cl}_{2}$ & - & -4.53 & -4.43 & -4.38 \\
\hline $\mathrm{Hf}_{3} \mathrm{~B}_{4}(\mathrm{OH})_{2}$ & - & -7.78 & -7.75 & -7.69 \\
\hline $\mathrm{Hf}_{3} \mathrm{~B}_{4} \mathrm{H}_{2}$ & - & -2.97 & -2.96 & -2.97 \\
\hline $\mathrm{Zr}_{3} \mathrm{~B}_{4} \mathrm{O}_{2}$ & - & -7.83 & -7.79 & -7.86 \\
\hline $\mathrm{Zr}_{3} \mathrm{~B}_{4} \mathrm{~F}_{2}$ & - & -6.43 & -6.43 & -6.42 \\
\hline $\mathrm{Zr}_{3} \mathrm{~B}_{4} \mathrm{Cl}_{2}$ & - & -4.69 & -4.63 & -4.67 \\
\hline $\mathrm{Zr}_{3} \mathrm{~B}_{4} \mathrm{H}_{2}$ & - & -2.99 & -2.98 & -2.97 \\
\hline $\mathrm{Zr}_{3} \mathrm{~B}_{4}(\mathrm{OH})_{2}$ & - & -7.89 & -7.87 & -7.84 \\
\hline
\end{tabular}

\section{REFERENCES}

1. Khazaei, M.; Ranjbar, A.; Esfarjani, K.; Bogdanovski, D.; Dronskowski, R.; Yunoki, S., Insights into Exfoliation Possibility of MAX Phases to MXenes. Phys. Chem. Chem. Phys. 2018, 20, 8579-8592. 